\title{
Epidural electrical stimulation of posterior structures of the human lumbosacral cord: 2. quantitative analysis by computer modeling
}

\author{
F Rattay $^{1}, \mathrm{~K}$ Minassian ${ }^{1}$ and MR Dimitrijevic*,2,3 \\ ${ }^{1}$ TU-BioMed, Vienna University of Technology, Austria; ${ }^{2}$ Department of Physical Medicine and Rehabilitation, \\ Baylor College of Medicine, Houston, Texas, USA; ${ }^{3}$ Ludwig Boltzmann Institute for Restorative Neurology and \\ Neuromodulation, Vienna, Austria
}

\begin{abstract}
Objectives: Analysis of the computed recruitment order of an ensemble of ventral and dorsal root fibers should enlighten the relation between the position of a bipolar electrode and the observed order of muscle twitches.

Material and methods: Thresholds of selected spinal root fibers are investigated in a two step procedure. First the electric field generated by the electrodes is computed with the Finite Element Method. In the second step the calculated voltage profile along each target neuron is used as input data for a cable model. For every electrode position the electrical excitability is analyzed for 12 large diameter ventral and dorsal root fibers of the second and fourth lumbar and first sacral segment. The predictions of the neural responses of any target fiber are based on the activating function concept and on the more accurate computer simulations of the electrical behavior of all nodes and internodes in the vicinity of the electrode.

Results: For epidural dorsal lumbosacral spinal cord stimulation we found the following rules. (i) The recruitment order of the spinal roots is highly related to the cathode level. (ii) Dorsal root fibers have the lowest threshold values, ventral root fibers are more difficult to excite and dorsal columns are not excitable within the clinical range of $10 \mathrm{~V}$. (iii) For a cathode close to the level of the spinal cord entry of a target fiber thresholds are lowest and spike initiation is expected at the border between cerebrospinal fluid and white matter; excitation of L4 roots is not possible with $210 \mu \mathrm{s} / 10 \mathrm{~V}$ pulses when cathode is more than $2.2 \mathrm{~cm}$ cranial to their entry level $(1.5 \mathrm{~cm}$ for $\mathrm{S} 1$ roots; standard data). (iv) Cathodes positioned (essentially) below the entry level cause spike initiation close to the cathode, in a region where the fibers follow the descending course within the cerebospinal fluid. (v) At rather low stimulation voltage twitches are expected in all investigated lower limb muscles for cathodes below L5 spinal cord level.

Conclusions: Our simulations demonstrate a strong relation between electrode position and the order of muscle twitches which is based on the segmental arrangement of innervation of lower limb muscles. The proposed strategy allows the identification of the position of the electrode relative to spinal cord segments.
\end{abstract}

Spinal Cord (2000) 38, $473-489$

Keywords: computer modeling; spinal cord stimulation; recruitment order; electrode position; lumbar spinal cord

\section{Introduction}

Clinical application of the electrical stimulation of the spinal cord with epidural electrodes cause a great variability in muscle responses including single twitches, constant tonus, suppression of spasms or generation of rhythmic activities. ${ }^{1-3}$ The shape of the electrodes and their positions relative to the excitable structures, the duration, intensity and polarity of the

*Correspondence: MR Dimitrijevic, Ludwig Boltzmann Institute for Restorative Neurology and Neuromodulation, Hofzeile 18-20, A-1190 Vienna, Austria stimulus signal as well as its repetition rate are the main elements that influence the large spectrum of those observed muscle activities which can be generated or affected by electrical stimulation.

The main purpose of this article is to find biophysical and neuro-physiological explanations for the recruitment order of muscle twitches as reported in the companion paper. ${ }^{2}$ However we have to simplify the situation in order to illuminate the relevant relations in the epidural electrical stimulation of the lumbosacral cord. We analyze which nerve structures 
become stimulated and the resulting sequence of excitation when the voltage of a stimulating dipole is increased.

Our study is based on the computer simulation of an ensemble of target neurons that are electrically excited by the stimulus signal. Usually such neural activities are simulated in a two step procedure. ${ }^{4-6}$ The first step is to compute the electric field generated by the electrodes, which is determined by the geometry of the various anatomical structures and their electrical conductivities. In the second step the calculated voltage profile along a target neuron is used as input data for an electric network model that represents the spatial form of the neuron. Analysis of the different substructures of a neuron confirms the suggestion that for most cases of electrically stimulated neurons with myelinated axons one node of Ranvier becomes the region of spike initiation. ${ }^{5,7,8}$ Therefore, we restricted the nerve model to the trajectory of axons and we applied a method that is similar to previous modeling of the electrically stimulated spinal cord. ${ }^{9-13}$

In a preliminary study we calculated the threshold values for posterior dorsal column fibers with $8 \mu \mathrm{m}$ axon diameter which we assume to be the largest column fibers within the lumbosacral region of the spinal cord. ${ }^{14}$ Fibers with smaller diameters are more difficult to activate. Calculated threshold for an $8 \mu \mathrm{m}$ dorsal column fiber with the smallest possible distance to a typical midsagittal electrode position (center of the electrode at the L2 spinal cord level) was $11.2 \mathrm{~V}$ for a biphasic $210 \mu$ s stimulus. There are only small threshold deviations for column fibers in the lumbosacral region if polarity is changed or monophasic pulses are applied. Because this threshold voltage is out of the $1-10 \mathrm{~V}$ range as applied in our clinical tests and because muscle responses in patients give no hints to dorsal column stimulation we have concentrated our study on $22 \mu \mathrm{m}$ diameter target fibers in the posterior and anterior roots.

The order of the calculated threshold values of spinal roots is related to the sequence of muscle responses in the muscle twitch experiments as described in the companion paper. $^{2}$ The muscle twitches are measured at stimulating frequencies of $5 \mathrm{~Hz}$. At such a low frequency each artificially generated action potential causes a single twitch. The single EMG potentials are of the same shape. From the point of theory we demonstrate effects of electrode positions with single monophasic and biphasic stimulus pulses because the shape and amplitude of the twitch potentials are independent from the repetition rate for low stimulation frequencies.

\section{Material and methods}

The assumptions about the electrodes and investigated pulse shapes are in accordance with the clinical application reported in the companion articles where the configuration with the maximum contact separa- tion of the Medtronic Quad electrode implant is activated. The two active contacts of the electrode, labeled as contact 0 and contact 3 , are assumed as cylindrical elements, each $3 \mathrm{~mm}$ high and $1.27 \mathrm{~mm}$ in diameter with a center to center separation of $27 \mathrm{~mm}$. Control measurements, eg of the Medtronic 3487 electrode driven by Itrel II stimulator in salt water both with $60 \quad \mathrm{Ohm}^{*} \mathrm{~cm}$ and with $500 \quad \mathrm{Ohm} * \mathrm{~cm}$ resistivity demonstrate that the pulse shape can be approximated as a rectangular pulse of $210 \mu \mathrm{s}$ that is followed by a long second phase of small amplitude which prevents charge accumulation. According to our investigations we conclude that this type of pulse shape will initiate spikes solely as responses to the first pulse and insignificant threshold changes are expected when the charge balanced situation is compared with the response from stimulation with monophasic rectangular pulses.

Our assumptions about a 'standard' shape of the spinal cord, its relative position to the vertebral bone, the trajectory of the rootlets and the other geometrical parameters of the body are based on the findings of Wall et $a l^{15}$ Kameyama et $a l^{16}$ and on the 'Body Explorer', ${ }^{17}$ an interactive program on the crosssectional anatomy of the 'Visible Human Male', with small modifications according to X-ray and MRI data of our patients (Figure 1). The electrical conductivities of the biological materials are listed in Table 1.

The patients were stimulated in a range from 1$10 \mathrm{~V}$ by $210 \mu$ s pulses. In the computer simulation the stimulation voltage was expanded over the clinical range in order to find a relation between the excitation thresholds for spinal nerve fibers and their distance to the electrode. Furthermore, we investigated the influence of rectangular monophasic pulses and different shapes of biphasic pulses on the excitation process of selected target neurons. In biphasic stimulation the first pulse was always rectangular. The second phase followed immediately supplying the same charge as the first pulse and was assumed to be either of rectangular shape or a function with exponential decay.

The electric field was computed with the finite element program ANSYS for a $24 \mathrm{~cm}$ transverse section of the human body (Figure 1) under quasistationary conditions, ie capacitive effects between the electrode and surrounding tissues are neglected when the electric field is calculated. ${ }^{20}$ However, the capacity of the neural membrane is included for computing the excitation of the target neurons. The boundary conditions are given by the voltages at the electrodes, and by the fact that no current flows out of the simulated volume.

The electric field was evaluated for selected trajectories representing the geometry of the myelinated axons of target neurons. In order to find the recruitment order of the roots of the spinal cord we simulated the excitation process in representative target fibers by a cable model with ion channel dynamics according to the model of Sweeney et al. ${ }^{21}$ 


\section{A}

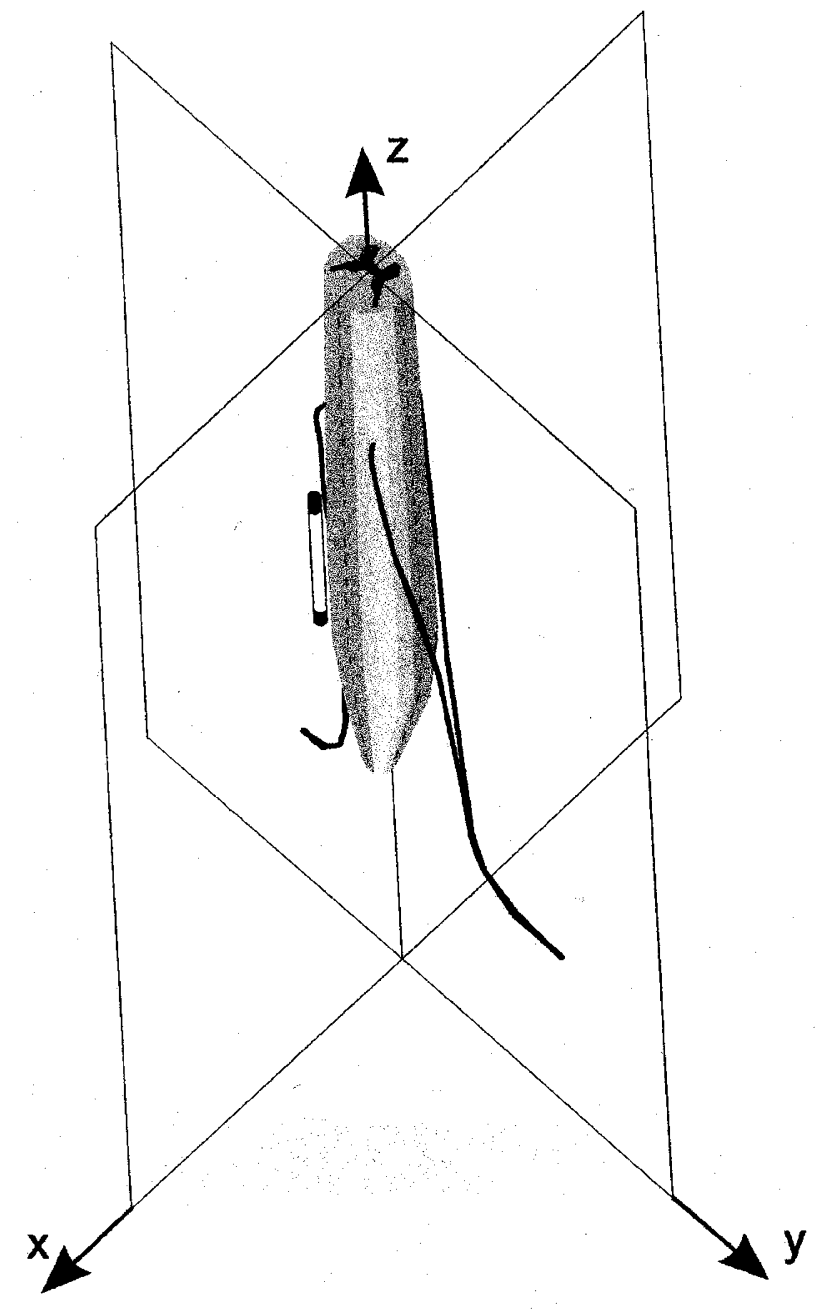

B
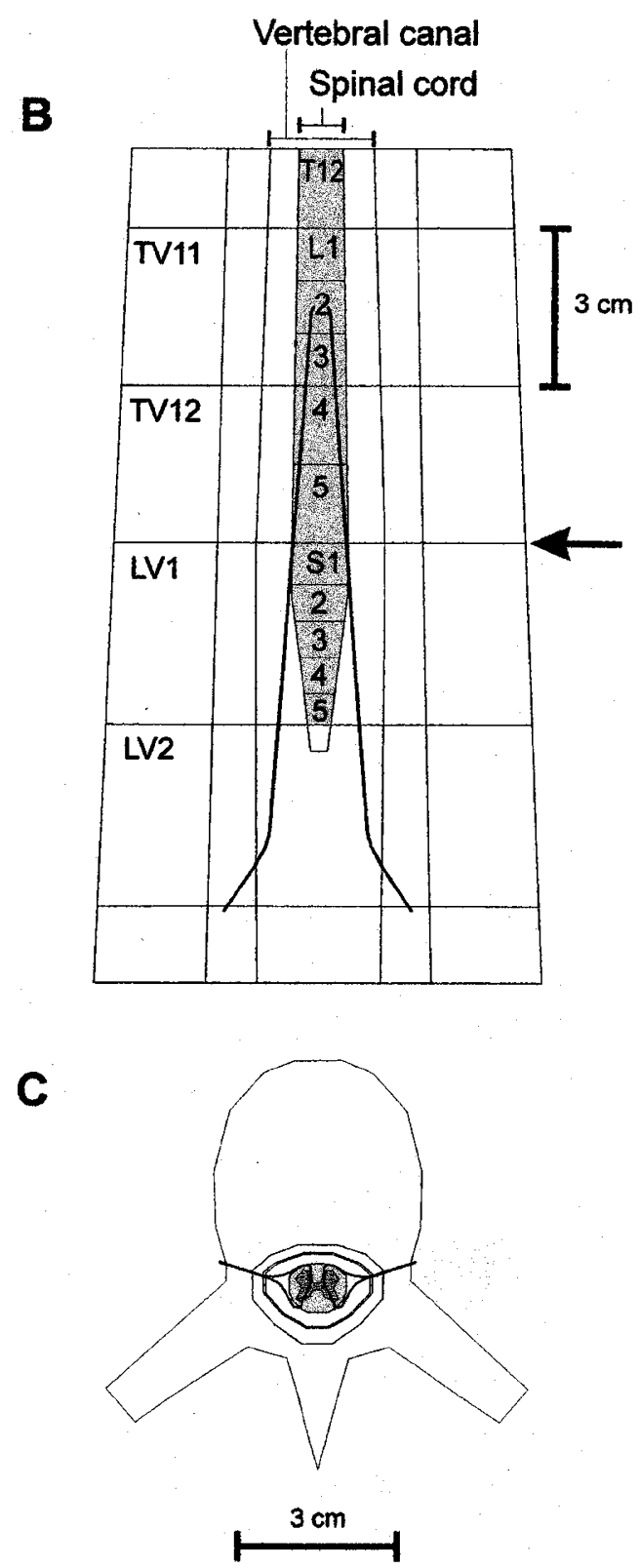

Figure 1 Geometry of the spinal cord with surrounding tissue and trajectories of L2 root fibers (dark lines) as used for finite element computation. (A) Position of a $3 \mathrm{~cm}$ long midsagittal electrode with the center at L4 spinal level. (B) Front view of spinal cord and simplified geometry of the vertebral column (TV thoracic vertebra, LV lumbar vertebra). The lumbar nerves as represented by L2 fibers have long spinal roots that descend within the dural sac to reach their appropriate vertebral level of exit. Arrow marks the position of the cross section as displayed in $(\mathbf{C})$. The cross section shows the boundaries between regions of different electrical conductivities: gray matter, white matter, cerebrospinal fluid, dura mater, epidural space, vertebral bone and surrounding tissue (comp. Table 1)

The activating function concept supported the computed results by theory. The fiber is represented as a compartment model where every node of Ranvier and every internode (myelinated part between two nodes) is simulated as a segment with individual electrical properties. For details see Rattay. ${ }^{4,5} \mathrm{We}$ used the following standard data for simulation: spinal root fiber diameter $\mathrm{D}=22 \mu \mathrm{m}$, axon diameter $\mathrm{d}=0.64 \mathrm{D}$, internodal length $100 \mathrm{D}$, nodal length $1.5 \mu \mathrm{m}$.

The first response of every node and every internode to a stimulus pulse can be estimated with the activating function, which quantifies the local change of membrane voltage. ${ }^{4,5,8}$ The activating function uses the extracellular voltage along the neuron as input data, but the complicated ion 
Table 1 Conductivities of the biological materials

\begin{tabular}{ll}
\hline Biological material & Conductivity $\left(\Omega . \mathrm{cm}^{-1}\right)$ \\
\hline Grey matter & $0.00025[\operatorname{Ref} 9,18]$ \\
White matter, transversal & $0.00083[\operatorname{Ref} 9,18]$ \\
White matter, longitudinal & $0.0072[\operatorname{Ref} 9,18]$ \\
Cerebrospinal fluid & $0.0167[\operatorname{Ref} 9,18]$ \\
Dura mater & $0.0003[\operatorname{Ref} 19]$ \\
Fat in the epidurial space & $0.0005[\operatorname{Ref} 9,18]$ \\
Vertebral bone & $0.00025[\operatorname{Ref} 9,18]$ \\
Surrounding layer & $0.0025[\operatorname{Ref} 9,18]$ \\
\hline
\end{tabular}

channel mechanisms are not required in order to find the position of strongest depolarization at stimulus onset and to obtain a rough estimation for the threshold value of the stimulus signal. For a regular myelinated nerve fiber the activating function is proportional to the second difference quotient of the external potential, ie the driving force is proportionate to the axial component of the gradient of the electric field. When a single stimulus pulse is applied to a fiber which has been in a resting state before, spike initiation usually is expected in the region with the highest positive values of the activating function. According to our assumptions of constant fiber diameter and constant internode lengths, the value of the activating function is related to the curvature of the extracellular voltage profile along the fiber. For a given stimulus intensity the curvature of the extracellular voltage profile is mostly influenced by (i) the distance to the electrode, (ii) the curvature of the roots and (iii) the change of electrical conductivity (cerebrospinal fluid - white matter) at the spinal cord entries of the spinal roots.

\section{Results}

Threshold as a function of electrode position and polarity for a single spinal root fiber

In a first example we demonstrate the influence of polarity on the threshold values of a posterior L4 root fiber. The midsagitally positioned electrode is moved in the rostro-caudal direction. In all cases shown in Figure 2 the entry of the fiber into the spinal cord is between the levels of the two contacts of the stimulating electrode. The excitation is always dominated by the position of the cathode. For the $0-3+$ configuration (active upper contact: 0 , active lower contact: 3) the L4 fiber will not be excited in the $10 \mathrm{~V}$ range when the cathode is more than $2.3 \mathrm{~cm}$ cranial to the entry level (Figure 2A). The specific form of the voltage/distance relationship shows rather low thresholds when the cathode is near the entry level and an extreme increase beyond $2 \mathrm{~cm}(50 \mathrm{~V}$ would be required at $3.1 \mathrm{~cm})$. This nonlinear phenomenon causes a sharp border for the excitation region (gray area in Figure 2A) and is a good indicator for determining the fiber entry level.
A

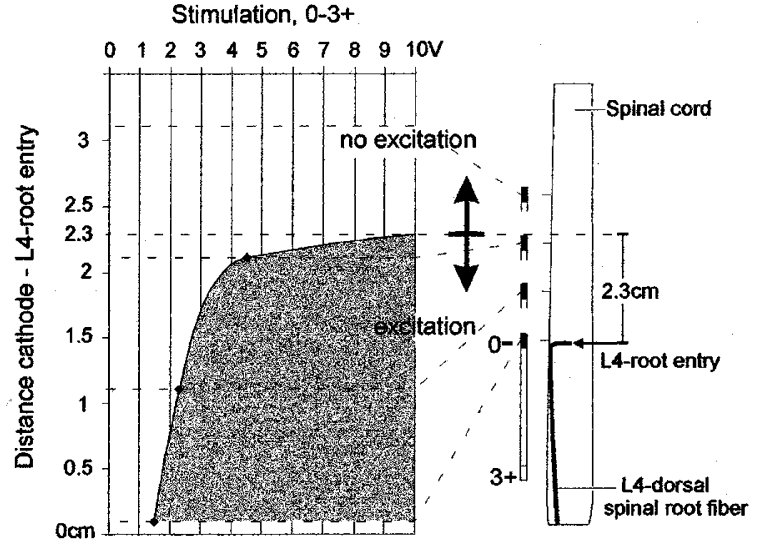

B

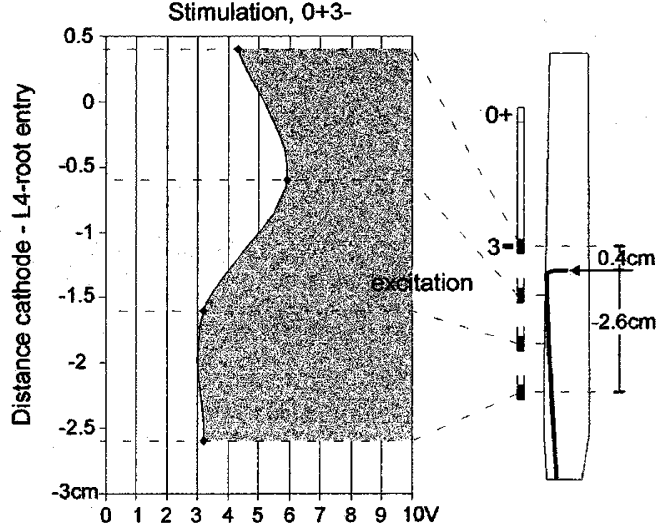

Figure 2 Excitation region of a posterior L4 root fiber. Threshold values are calculated for four electrode positions, polynomial interpolation for other positions. Positions of the electrode with the black marked cathode, L4 spinal root fiber and spinal cord from a side view (right side). The threshold function pictures are zoomed according to the dashed lines

When polarity is changed $(0+3-)$ the L4 root fiber will always be excited with low or medium threshold values (Figure 2B). Here the cathode is always close to some excitable region of the fiber. Because of the caudal course of the posterior root trajectory the excitability is not much changed when the electrode is moved several $\mathrm{cm}$ caudal to the entry point. All posterior fibers arising from the L4 segment come close together on both sides and therefore all large diameter L4 fibers have similar thresholds for the low positioned electrode. Similar fiber behavior is expected for other lumbosacral spinal levels.

\section{Stimulation with monophasic rectangular pulses}

The excitation process and the influence of polarity on threshold voltage is easy to explain when a single rectangular stimulus is applied. The trajectory of the next investigated target neuron passes both contact levels of the electrode, which describes the situation for a L2-dorsal root fiber with the electrode positioned as indicated in Figure 3. Figure 3 demonstrates the 
relations between extracellular voltage, activating function and the membrane voltages resulting in a propagating action potential when stimulated just above threshold. In case $\mathrm{A}(0-3+)$ the potential of the upper contact (cathode) is $-2.9 \mathrm{~V}$ with respect to the anode, whereas change of the polarity has a higher threshold value of $5 \mathrm{~V}$ (case $\mathrm{C}$ ). This effect can be explained in the following way: The curved trajectory of the nerve fiber causes some asymmetry in the extracellular voltage profile. There is stronger curvature at the upper contact (cathode in case $\mathrm{A}$ is at a position with length coordinate $\mathrm{s}=1.4 \mathrm{~cm}$ along the $\mathrm{L} 2$ posterior target fiber) than at the lower contact (anode in case $A$ at $s=4.4 \mathrm{~cm}$ ). Therefore the maximum value of the activating function $f$ is larger than its minimum value. As mentioned above, the value of the activating function represents the temporal membrane voltage change at stimulus onset, ie it defines the slope in every membrane voltage curve (lines at the right part of Figure 3) at the beginning of the stimulating pulse. The arrow in Figure 3A marks the maximum value of $f$ and - because the strongest slope value causes the quickest rise of membrane voltage - the arrow marks also the place of spike initiation for supra-threshold stimulation. Comparison of the first part of every line of the membrane voltages in Figure $3 \mathrm{~A}$ shows that the negative excursions are always smaller than the positive ones (arrow). Changing the polarity of case A therefore results in a subthreshold response (Figure 3B). Reversed polarity $(0+3-)$ needs $72 \%$ higher stimulus to reach threshold and the spike initiation site has moved to the $4 \mathrm{~cm}$ region (Figure $3 \mathrm{C}$ ).

If both active poles of the implant are caudal to the origin of a spinal root fiber we have to expect a similar situation as in Figure 3, ie both poles contribute significantly - with alternating polarity - to the extracellular voltage profile along the fiber. Quite different is the 'unipolar' voltage profile for a fiber which originates caudal to the dipole (Figure 4). By comparison of Figure $3 \mathrm{C}$ and $4(5 \mathrm{~V} / 210 \mu$ s stimulus in both cases) the first $4.9 \mathrm{~cm}$ influence on the extracellular voltage in S1 fibers is lost (see also Figure 5). This has minor effect for positive stimuli $(0+3-)$. However, change of polarity causes quite different results when the influence of one contact is lost. With negative monophasic stimuli we cannot excite the S1 fibers with voltages below $100 \mathrm{~V}$, because this fiber has a small negative part of the activating function at the first nodes only (Figure 4). The polarity effect of the electrode level is shown in Figure 5. Furthermore, the three main components that influence the curvature of the extracellular potential are explained in more detail.

This analysis demonstrates that the unipolar excitability is a good indicator for the position of the electrode. The level of every spinal root fiber entry relative to the electrode is fundamental for the profile of the extracellular voltage. For most neurons the asymmetry of the voltage profile is distinct enough to cause different thresholds for positive and negative polarity. The influence of stimulus polarity on threshold has two extreme cases: (i) a bipolar voltage profile with similar positive and negative maximum values and similar curvature near the contacts causes nearly identical thresholds for cathodic and anodic stimuli and (ii) a unipolar voltage profile causes extreme differences in cathodic and anodic threshold values.

Electrode voltages that excite the target fibers in the dorsal and ventral roots are shown in Figure 6 as black bars for four midsaggital electrode positions in different caudal levels. The following results are typical (Figure 6): (i) Dorsal root fibers have lower thresholds than ventral ones. (ii) Every target fiber has its minimum threshold value when the cathode is positioned at the level of its entry. (iii) The cathode is the dominant stimulating element but the position of the second contact has also an influence. As an example, the $(0+3-)$ case in Figure 6A has higher L2 thresholds although the cathode position is nearer to the L2 fiber entry than in the $(0-3+)$ case in Figure 6D. (iv) With the cathode positioned at S1 level all simulated dorsal roots are excitable within the clinical range of $10 \mathrm{~V}$ (Figure 6D left) because the L2 and L4 fibers pass the cathode level within the dural sac to reach their vertebral level of exit. (v) The recruitment order of the roots of different spinal levels is characteristic for every cathode level. Thus, the recruitment order which is related to the sequence of the muscle twitch responses is a proper tool to detect the approximate position of the electrode. (vi) Roots arising caudal of the electrode have an activating function shaped like in Figure 4. Therefore they are only excitable with the $0+3-$ configuration. Observing these differences of the recruitment order when polarity is changed provide us with an accurate tool to identify the electrode level. The level of the lower contact of the electrode is defined in the following way: all roots which are easily excitable by the $0+3-$ configuration and not excitable by the other polarity $0-3-$ originate caudal to the lower contact of the electrode.

\section{Biphasic stimulation}

In every stimulation cycle a second phase is applied for charge compensation. However, the second phase has minor stimulating effect if it is of small amplitude and long duration. Our clinical data are derived from biphasic stimulation with such a small second phase amplitude that its stimulating influence can be neglected and thus the results correspond to the monophasic responses (Figure 6).

We demonstrate the influence of the second phase shape for an S1 posterior root fiber and a midsagittal positioned electrode at L4 spinal level stimulating with three different biphasic signals (Figure 7). For this example which corresponds to Figure 4, the shape of the activating function implies that excitation can only be initiated by the positive phase of a pulse. For the $0+3-$ configuration the 

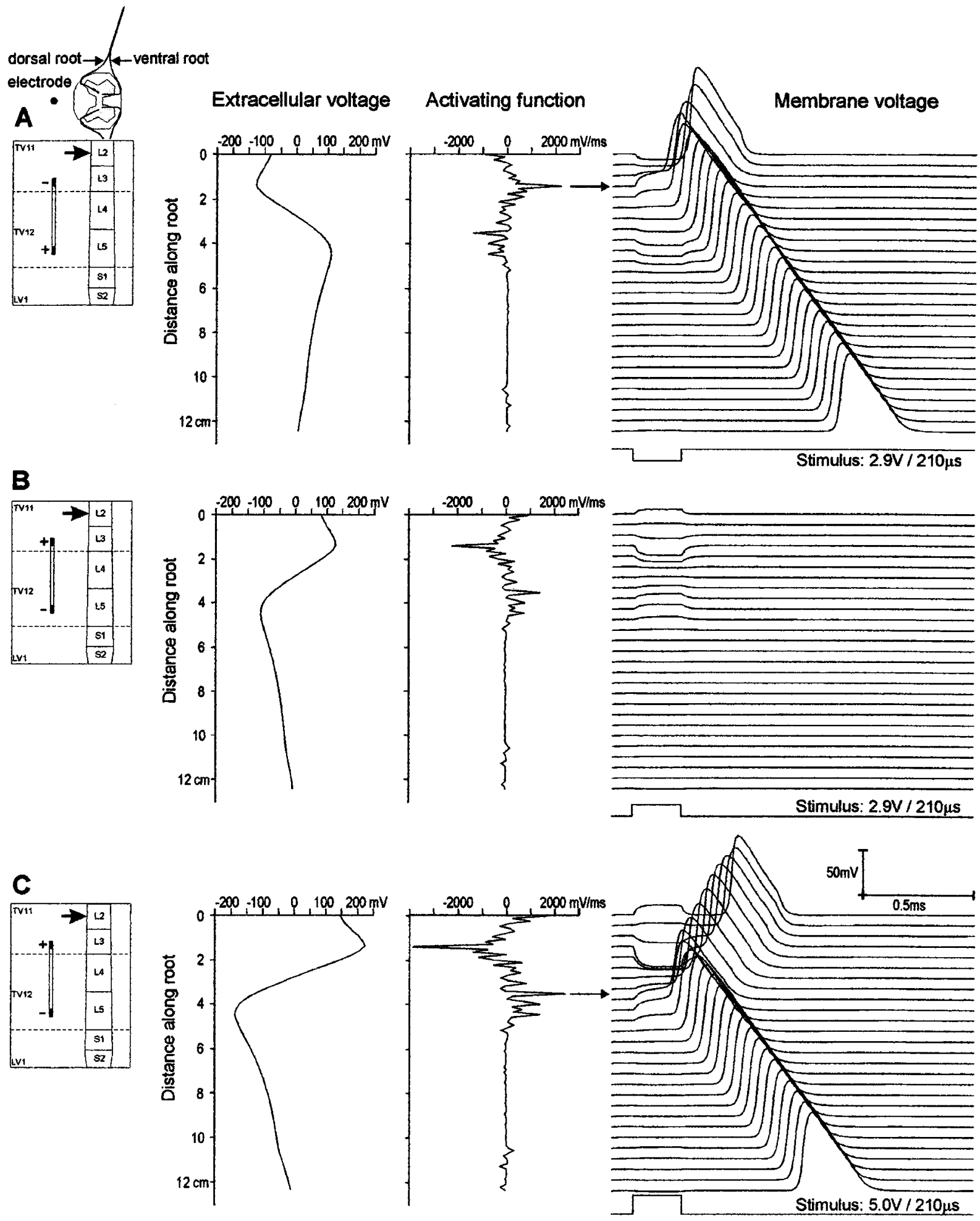

Figure 3 Input data (extracellular voltage) and excitation patterns for a dorsal L2 fiber stimulated with a midsagittal electrode at L4 spinal level (center of the dipole is $2.5 \mathrm{~cm}$ caudal to the L2 root, left pictures). Extracellular voltage and the activating function are displayed as functions of fiber's length coordinate - starting point is within the spinal cord. Extracellular voltage changes insignificantly from compartment to compartment, but the activating function has a zig-zag shape because of variations in the nodal and internodal influences (in general the larger values belong to the nodes; for details comp. Eq. 3 in Rattay ${ }^{5}$ ). Every line of the membrane voltage pictures shows the temporal evolution at a node of Ranvier, the responses of every second node and of all internodes are not displayed. (A) Excitation with negative threshold stimulus $(-2.9 \mathrm{~V}$, active poles: $0-3+)$ ie the standard polarity is changed and the upper contact which corresponds to a path-length of $1.4 \mathrm{~cm}$ becomes the cathode of the 
excitation is caused by the first phase. The threshold values are $4.8 \mathrm{~V}$ for all three cases, regardless whether the pulse shape of the second phase is rectangular or an exponential function (Figure 7, left side). However, for the $0-3+$ configuration the threshold voltages essentially depend on the shape of the second phase (Figure 7, right side). Stimulating with a rectangular phase which means a constant amplitude for $210 \mu \mathrm{s}$ is more effective (threshold $4.5 \mathrm{~V})$ compared to signals with exponential decay. The decay and the ratio of the amplitudes of phases one and two is defined by the time constant. For greater time constant values of the second phase higher threshold voltages are needed: Time constants

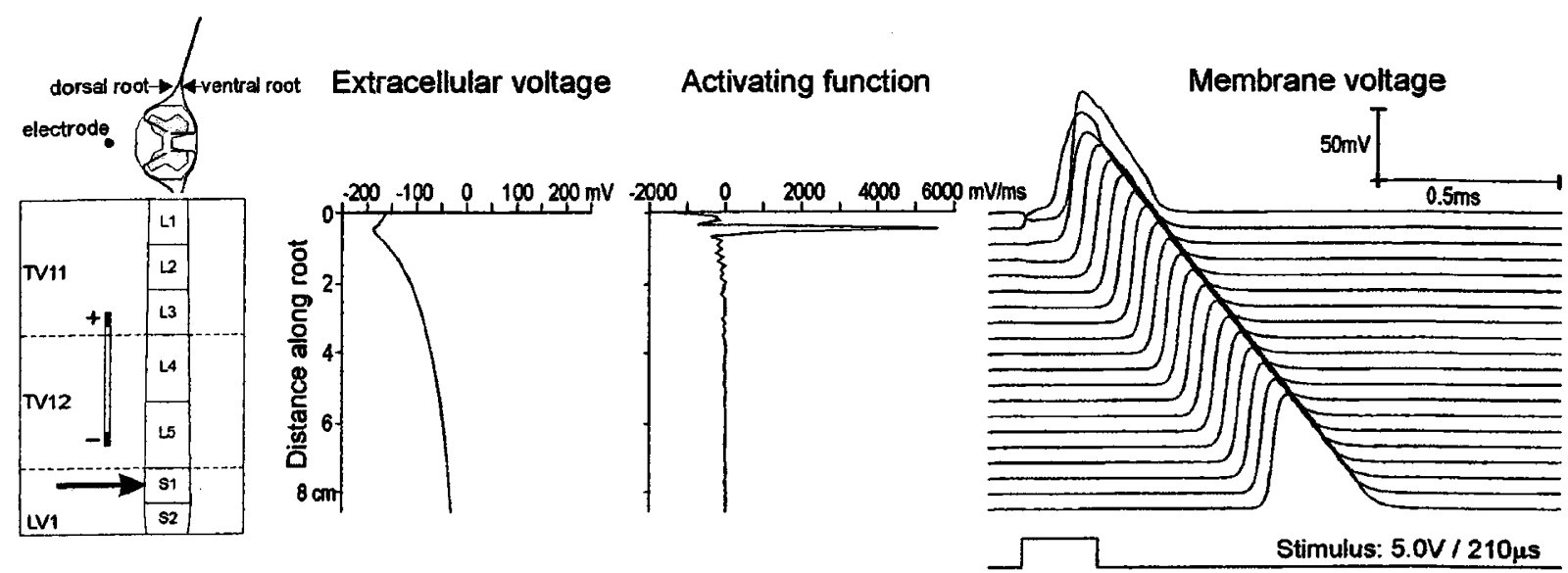

Figure 4 Input data and excitation patterns for a dorsal S1 fiber stimulated 4\% above threshold with a midsagittal electrode at L4 spinal level (same position as in Figure 3). Note, that the positive influence of the activating function is restricted here to a single node, whereas it is distributed to three nodes in Figure $3 \mathrm{C}$, which is a consequence of the deviation in the neural trajectories

\section{Region with the smallest distance of the L2-root to the lower electrode}

Region of the S1-root entry into the spinal cord, strong curvature because of (ii) the strong bending of the spinal root and (iii) large differences in the electrical conductivities of the cerebrospinal fluid and the white matter

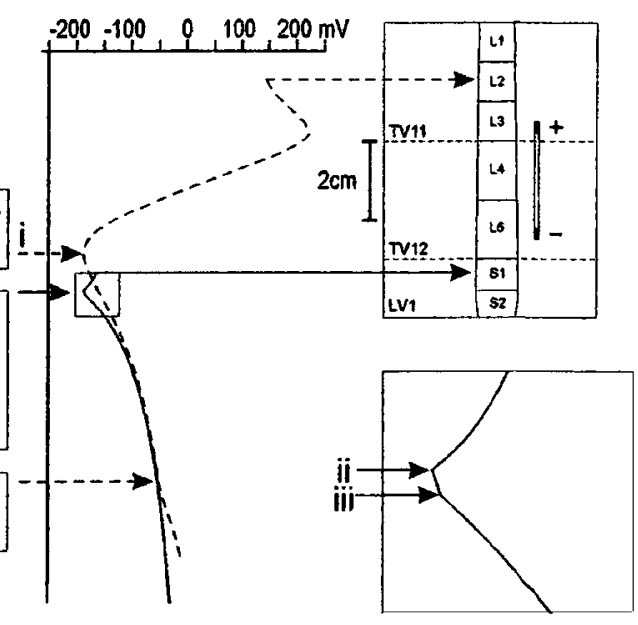

Figure 5 Comparison of the extracellular voltage along a L2 fiber (dashed line) and a S1 fiber (full line) as shown in Figures $3 \mathrm{C}$ and 4 . Because the $\mathrm{S} 1$ fiber origin is about $0.95 \mathrm{~cm}$ caudal to the lower electrode and $4.9 \mathrm{~cm}$ below the $\mathrm{L} 2$ entry the influence of the positive electrode on S1 is rather small. The extracellular potential within a part of the trajectories of both fibers is nearly identical. Note that differences in curvature (indicated by arrows and numbers i-iii) result from different influences

dipole. Note that the values of the activating function defines the slopes of membrane voltage at stimulus onset. Arrow marks the maximum value of the activating function and the position of spike initiation. (B) Change of polarity $(2.9 \mathrm{~V}$, active contacts: $0+3-$; subthreshold response) causes mirror pictures of the voltage profile, the activating function and the membrane voltages in the first part of every line (right). (C) Threshold increases to $5 \mathrm{~V}$ for the $0+3-$ configuration; spike generation at a more caudal position of the root relative to the region in (A) 


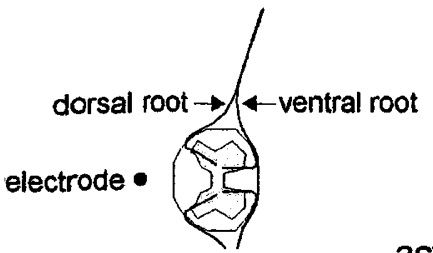

A
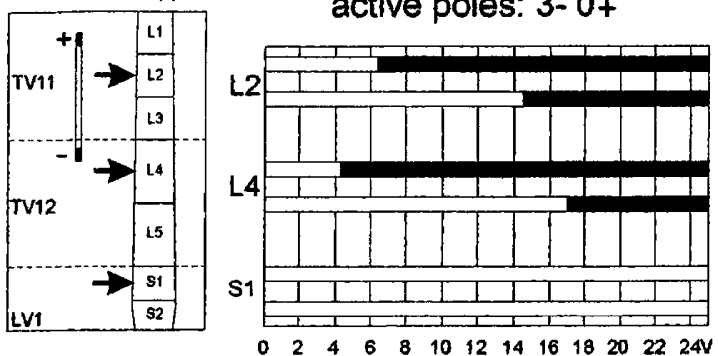

B
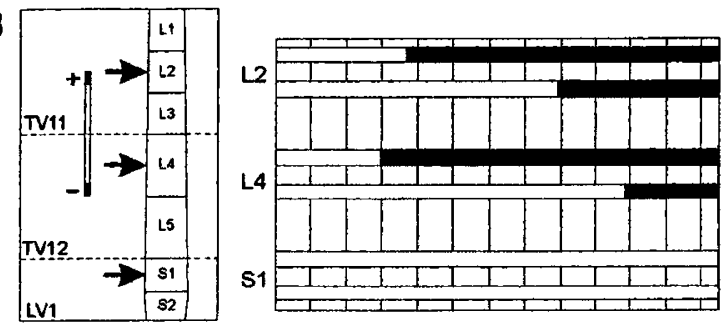

$\begin{array}{llllllllllllll}0 & 2 & 4 & 6 & 8 & 10 & 12 & 14 & 16 & 18 & 20 & 22 & 24 \mathrm{~V}\end{array}$

C
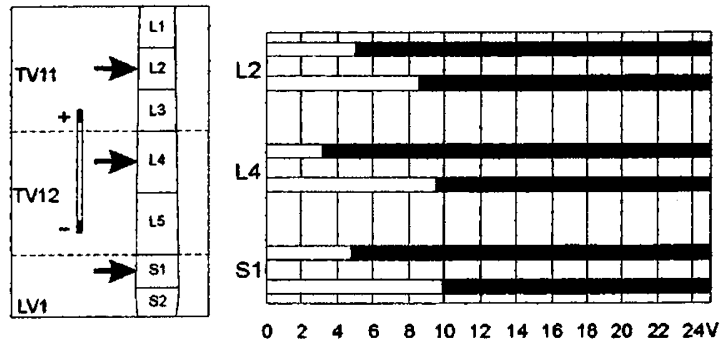

D

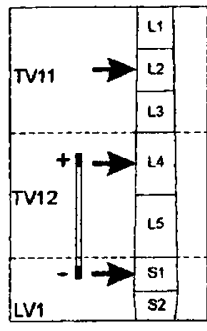

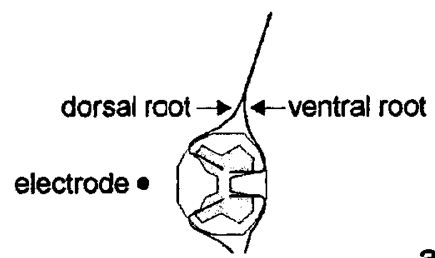
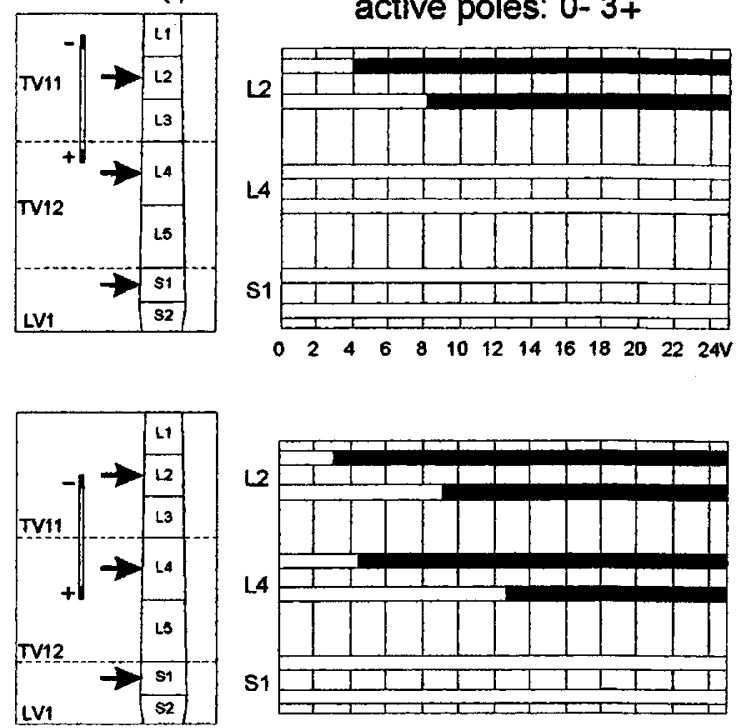

$\begin{array}{llllllllllllll}0 & 2 & 4 & 6 & 8 & 10 & 12 & 14 & 16 & \text { t8 } & 20 & 22 & 24 \mathrm{~V}\end{array}$
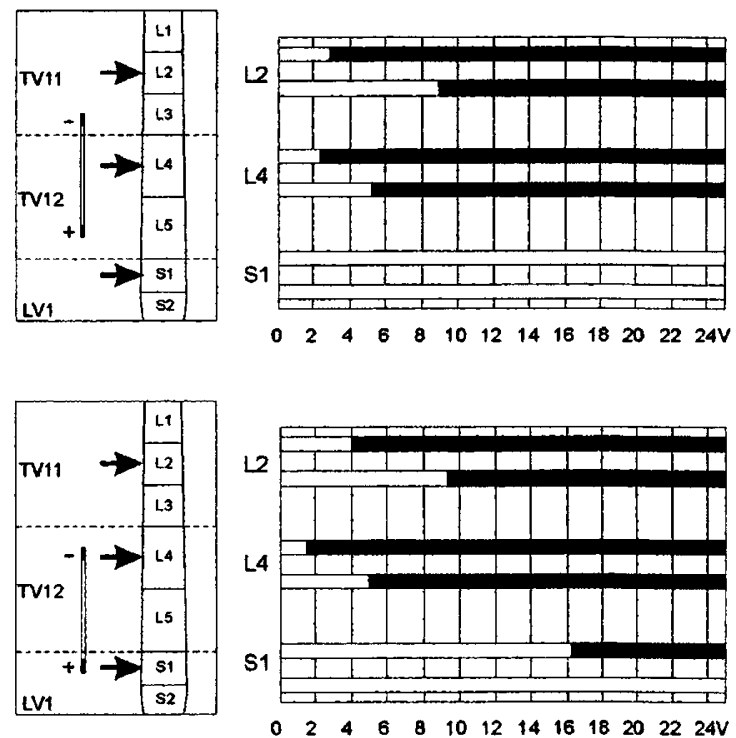

Figure 6 Activation of spinal root fibers for midsagittal electrodes positioned at different spinal levels with single rectangular $210 \mu \mathrm{s}$ pulses. The left pictures show the position of the electrode relative to the spinal cord segments. Arrows mark the entries into the spinal cord of the six simulated target fibers which are evaluated in the corresponding right pictures. The upper and the lower black bar of each segment marks the simulated excitation range of the dorsal and ventral roots, respectively. Responses of left and corresponding right root fibers are the same because of symmetry. From A to D the electrode is moved in caudal direction in $1 \mathrm{~cm}$ steps. The excitation threshold of every target fiber increases drastically when cathode is moved cranially far away from the fiber's entry into the cord. Polarity: $0+3-$ on the left side and $0-3+$ (right). Note, that in clinical application the highest applied voltage was $10 \mathrm{~V}$

of $210 \mu \mathrm{s}$ and $420 \mu \mathrm{s}$ cause thresholds of $6 \mathrm{~V}$ and $10.4 \mathrm{~V}$, respectively. Time constants of our Medtronic equipment were of the order of $4 \mathrm{~ms}$. In this case excitation of an S1 fiber with the second phase needs amplitudes one order higher than the clinical $10 \mathrm{~V}$ range. 

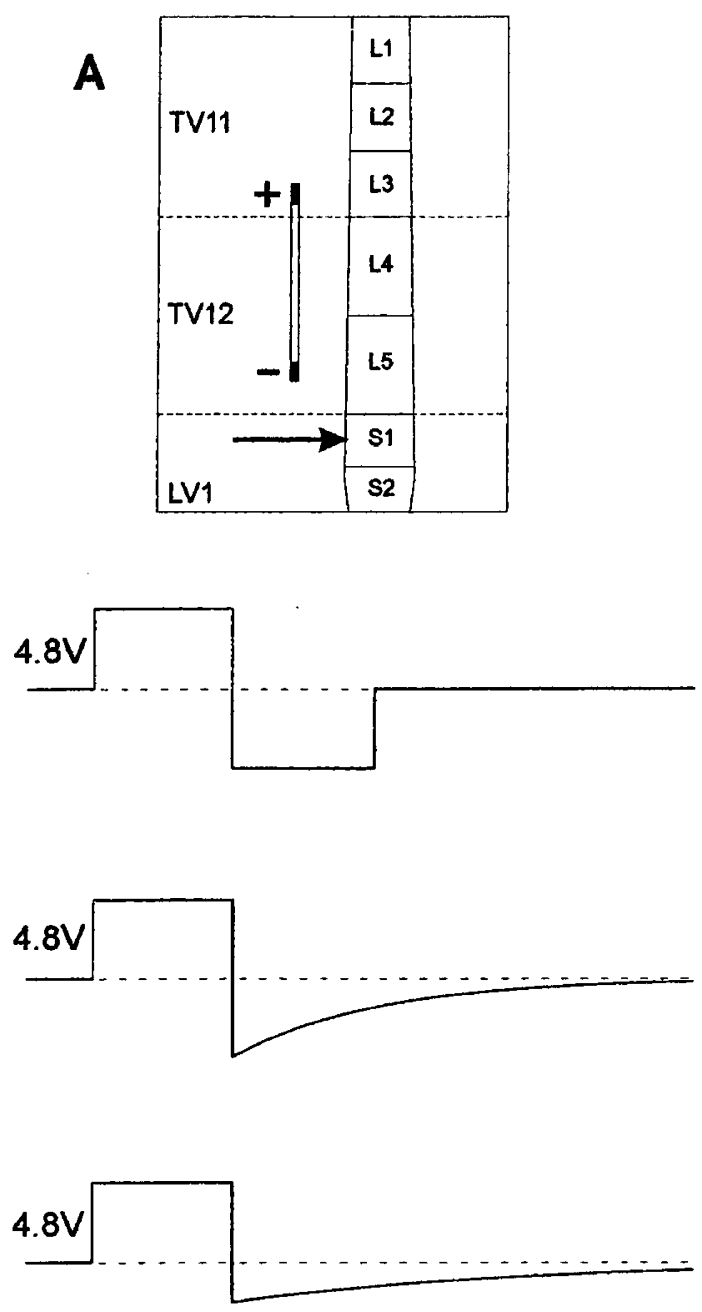

B
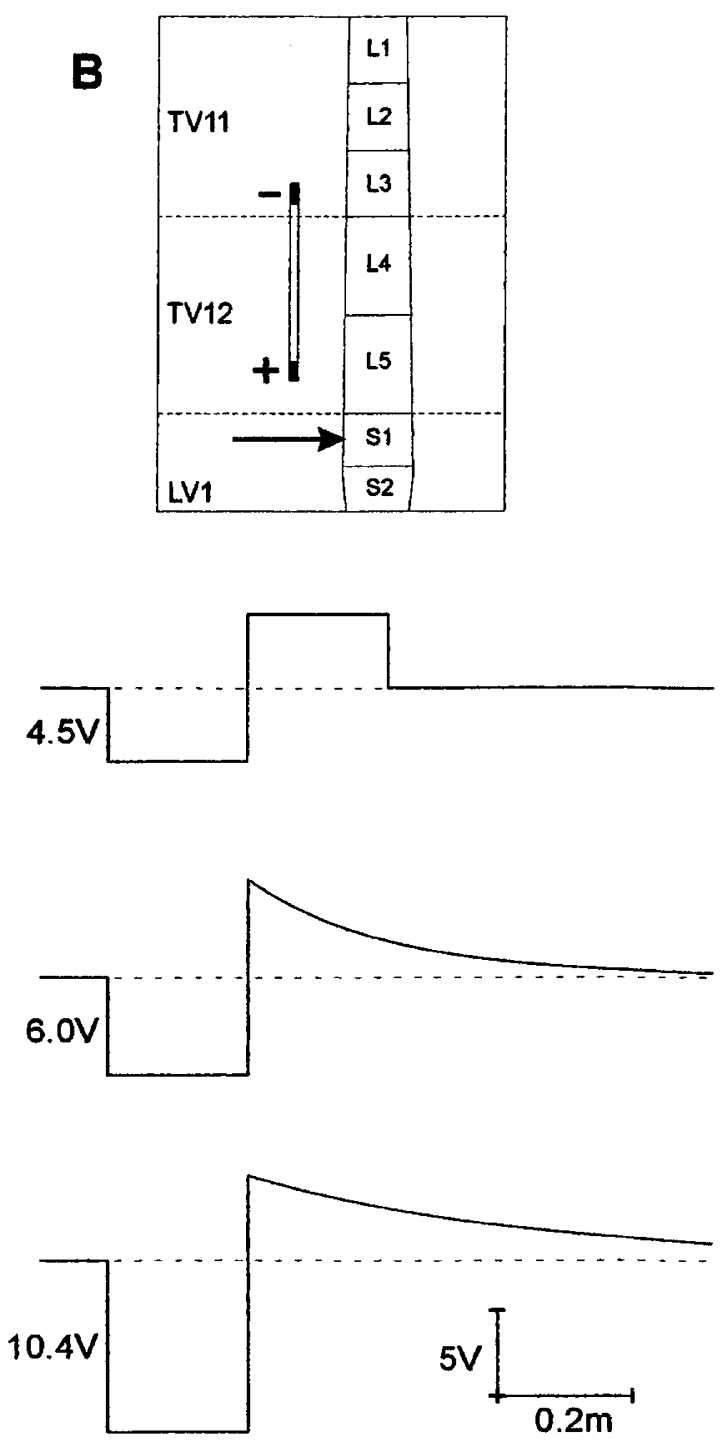

Figure 7 Threshold voltages for biphasic stimulation of an S1 posterior root fiber with a midsagittally positioned electrode at L4 spinal level. For the computer simulation we assumed a constant voltage-current relation at the stimulating electrodes. With this assumption the charge balance condition demands equal areas for the first and second phase. The duration of the first phase is always $210 \mu \mathrm{s}$. Second phase in case (A) is rectangular $(210 \mu \mathrm{s})$. In (B) the compensating phase has an exponential decay with a $210 \mu$ s time constant which causes same amplitudes for the first and second phase, whereas in (C) doubling of the time constant results in a 2:1 relation of the pulse amplitudes for charge balance. Note that the negative first phase of the pulse (A right) causes easier excitation than starting from the resting state of the membrane voltage (A left)

Both polarities $(0+3-$ and $0-3+)$ have similar threshold values if the stimulus signal consists of two rectangular phases of the same duration and amplitude (Figure 7A). For a given stimulus strength two groups of fibers are stimulated: One group is stimulated by the contact which act as the cathode during the first phase; when polarity is changed additional fibers become excited by the other contact which is then the cathode. This behavior can generally be expected for midsagitally (Figure 8) as well as for laterally positioned electrodes (Figure 9). Comparisons of corresponding cases in Figures 6 and 7 show smaller thresholds for some fibers when stimulated with biphasic rectangular pulses. These fibers profit by being closer to the cathode during the second phase. In the monophasic case (Figure 6) these fibers have the smaller distance to the positive contact of the electrode.

For posterior electrode positions the dorsal roots are usually easier to excite than the ventral ones. This fact is demonstrated in Figures 6 and 8 where the lower black bars (corresponding to the ventral roots) are shorter for every calculated root level. Electrodes 

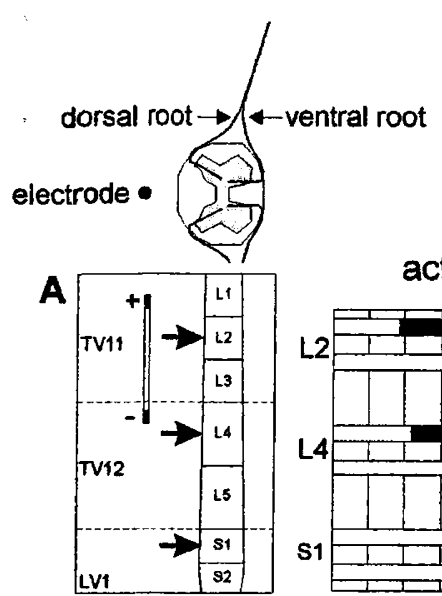

active poles: $3-0+$

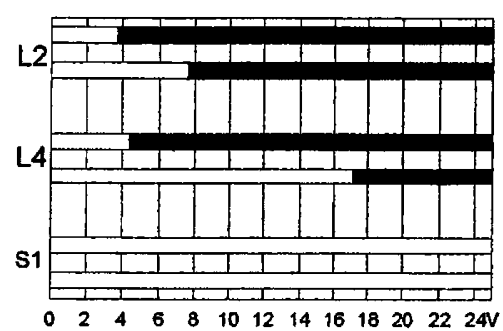

$\mathbf{B}$
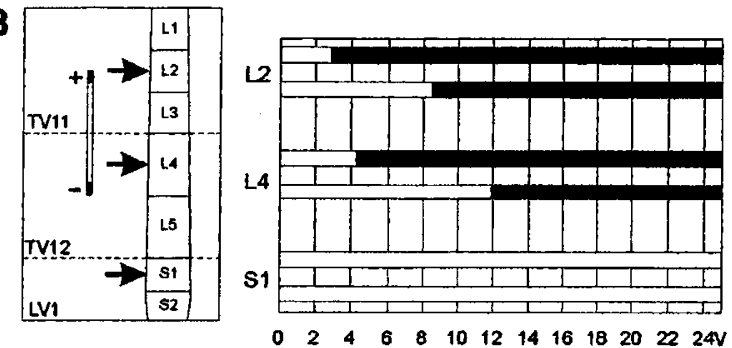

C
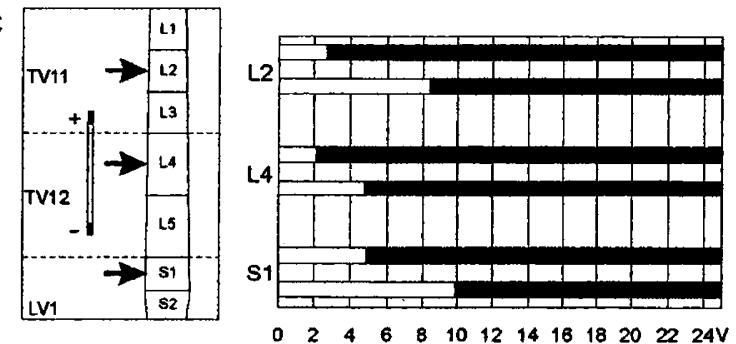

D

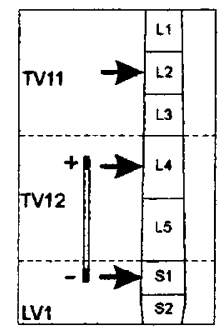

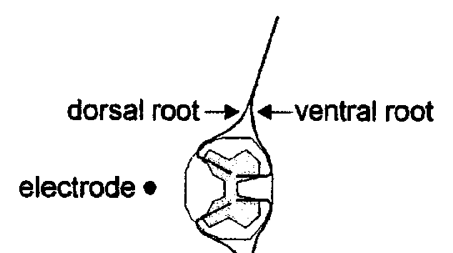

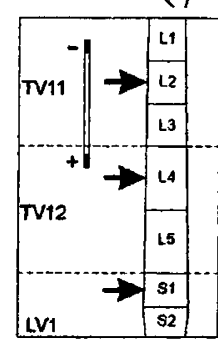

active poles: $0-3+$
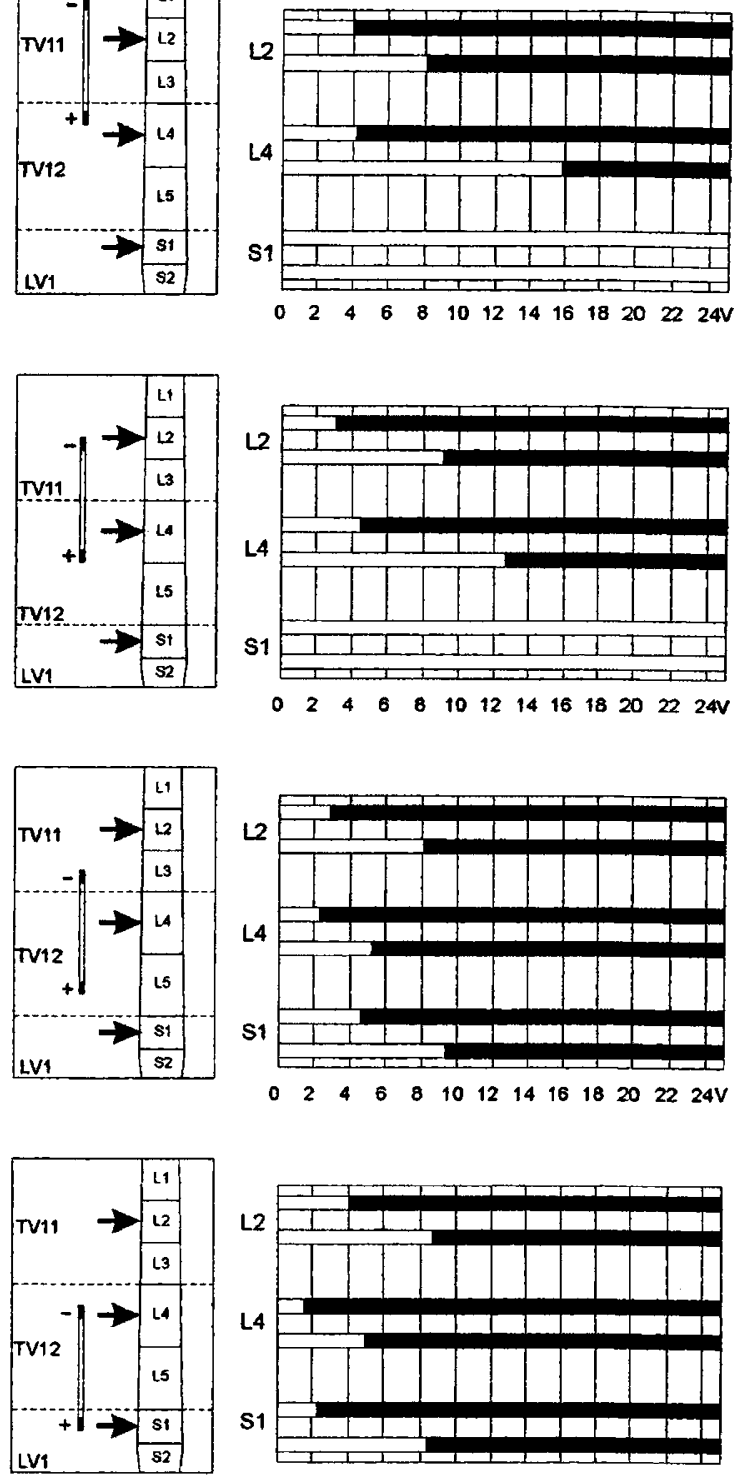

$\begin{array}{lllllllllllll}0 & 2 & 4 & 6 & 8 & 10 & 12 & 14 & 16 & 18 & 20 & 22 & 24 \mathrm{~V}\end{array}$

Figure 8 Activation of spinal root fibers for midsagittal electrodes positioned at different spinal cord levels with biphasic rectangular $210 \mu \mathrm{s}$ pulses. Same geometry as for the monophasic case in Figure 5. The length of every black bar is a combination of the maximum values of the two corresponding monophasic situations $(0+3-$ and $0-3+)$. Additionally, the effect mentioned in Figure 7A (starting not from the resting state for phase 2) lowers the threshold for all cases where the nerve is excited with the second phase. This effect explains also the small deviations between the $0+3-$ and the $0-3+$ cases when changing the polarity (left and right)

with an extreme lateral position can lead to a different result. Figure 9 shows an example where the ventral roots at the left side of the patient are closer to the electrode and therefore they respond to smaller stimuli.

\section{Discussion}

Characteristics of the lumbosacral cord

Our modeling study demonstrates that epidural stimulation of the lumbosacral cord with electrodes in 


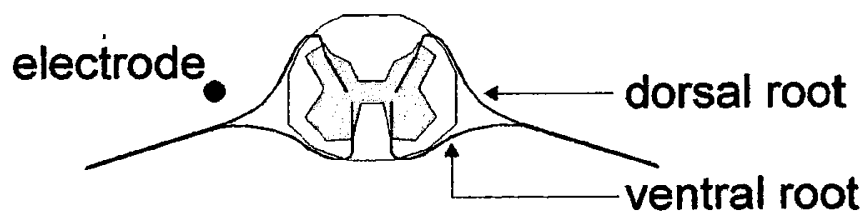

A

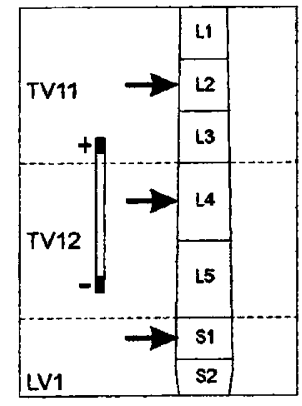

B

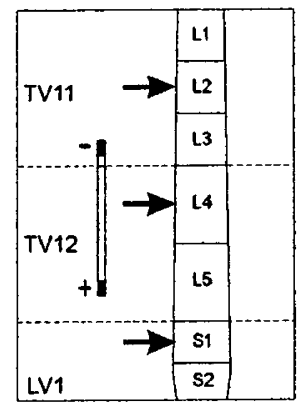

right side

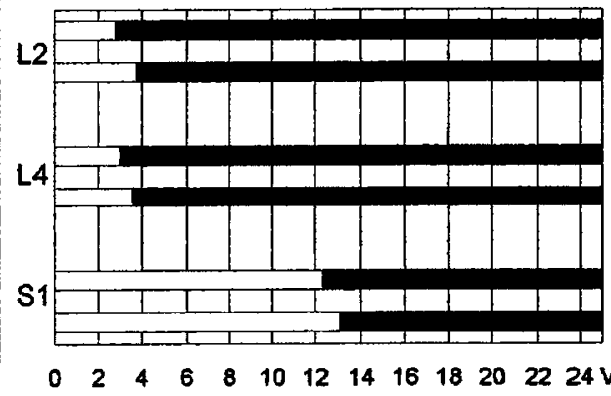

left side

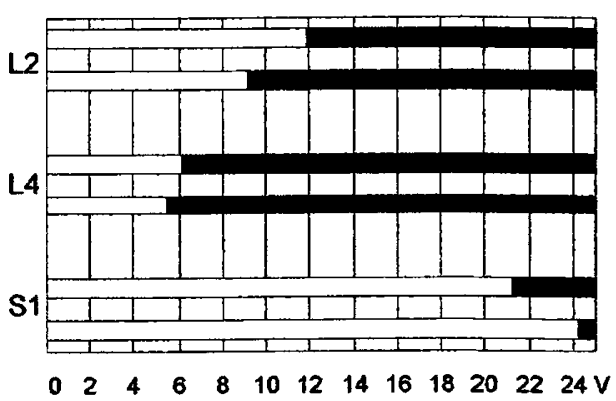

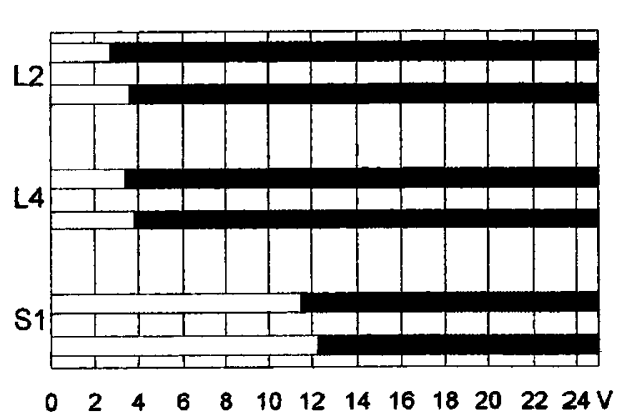

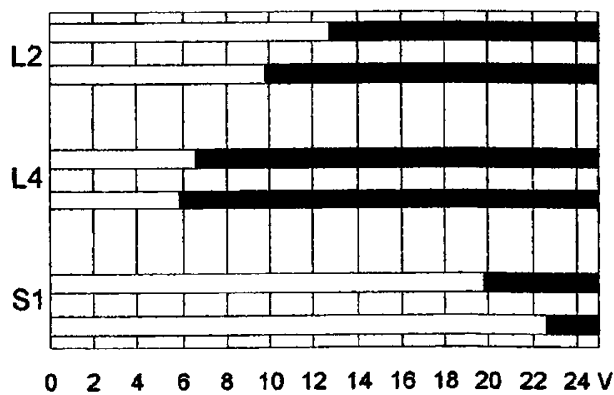

Figure 9 Activation of spinal root fibers for an extreme lateral electrode position with biphasic $210 \mu$ s pulses. Situation as in Figure $8 \mathrm{C}$ but with the electrode moved to the right side of the patient; therefore the result is asymmetric: the fibers of the left side have higher thresholds. Note that in this exceptional case the distance between the electrode and the regions of spike initiation at the left side is smaller for the ventral roots and therefore the threshold values of these fibers are lower than those of the dorsal roots

the posterior position activates the large diameter sensory fibers of posterior roots first, when the voltage is gradually increased. Stronger stimuli are needed to excite even the thickest motor neurons of the anterior roots. (Note however a reversed recruitment order for the roots at the opposite site of the electrode if the electrode is in an extreme lateral position Figure 9). The calculated minimum threshold value of dorsal column fibers in the lumbosacral region is $11.2 \mathrm{~V}$. Clinical observations also suggest that dorsal root fibers have the smallest threshold and dorsal column fibers are stimulated only in exceptional cases when voltages close to $10 \mathrm{~V}$ are applied.

The lumbosacral cord levels essentially differ from the corresponding vertebral levels with remarkable individual variability. In caudal direction the spinal cord diameter gradually grows to form the lumbar enlargement, then tapers and terminates between the first and second lumbar vertebral segment. The specific structure of the lumbosacral cord requires a three dimensional model with altering cross-sections which is a different approach compared to the prismatic geometries studied with the finite element technique by Coburn and $\operatorname{Sin}^{9}$ and to the finite difference method of Holsheimer and coworkers. ${ }^{11-13}$

For epidural stimulation the region of spike initiation is not restricted to the fiber entry into the spinal cord. As an example L2 posterior root fiber threshold is $3 \mathrm{~V}$ for $210 \mu$ s monophasic pulses when the cathode is at the L2 cord segment level. The same fiber can still be stimulated for a cathode positioned $5 \mathrm{~cm}$ caudal to the L2 level with $7 \mathrm{~V}$, while excitation is not possible when cathode is more than $2.5 \mathrm{~cm}$ above.

The level of the electrode with respect to the spinal cord is of high importance for several clinical applications, especially in order to reduce spasms ${ }^{3}$ and to activate the spinal cord locomotion pattern generator. ${ }^{1}$ Usually the electrode position is defined relative to vertebral segments but in living humans the location of the corresponding spinal cord segments cannot be identified by currently available image techniques. A great variance in spinal cord segment lengths has to be considered when simulated results 
are interpreted (Table 2). Our simulations are based on a geometry as shown in Figure 10A which is in some contradiction with the segment levels derived from Lang and Geisel data ${ }^{22}$ (Figure 10B).

Muscle twitch measurements are used as a functional method to get information about the electrode position as reported in the first article of this series. Note however, that the different muscle groups are innervated by several segments and thus there is no direct relationship between EMG responses and excited cord segments, especially for low positioned electrodes.

Table 2 Mean values and ranges of spinal cord segment lengths in mm according to different authors. Gray shaded values are used in Figure 10B to construct a relation between vertebral and cord levels

\begin{tabular}{lcccc}
\hline & $\begin{array}{c}\text { Lang }^{\text {and Geisel }} \\
\text { Old people }\end{array}$ & $\begin{array}{c}\text { Lang and Geisel } \\
\text { Young people }\end{array}$ & Diem $^{23}$ & Luderitz $^{24}$ \\
\hline L1 & 10.33 & $13.45(8-24)$ & 15 & $13.75-15.9$ \\
L2 & 9.88 & $12.0(8.5-21)$ & & $10.5-13.75$ \\
L3 & 8.75 & $11.0(7-21)$ & 9 & $7.5-10.25$ \\
L4 & 7.78 & & & $5.5-7.5$ \\
L5 & 6.29 & & 8 & \\
S1 & 6.89 & & & \\
S2 & 6.39 & & & \\
S3 & 5.41 & & & \\
S4 & 3.88 & & & \\
S5 & 2.32 & & & \\
\hline
\end{tabular}

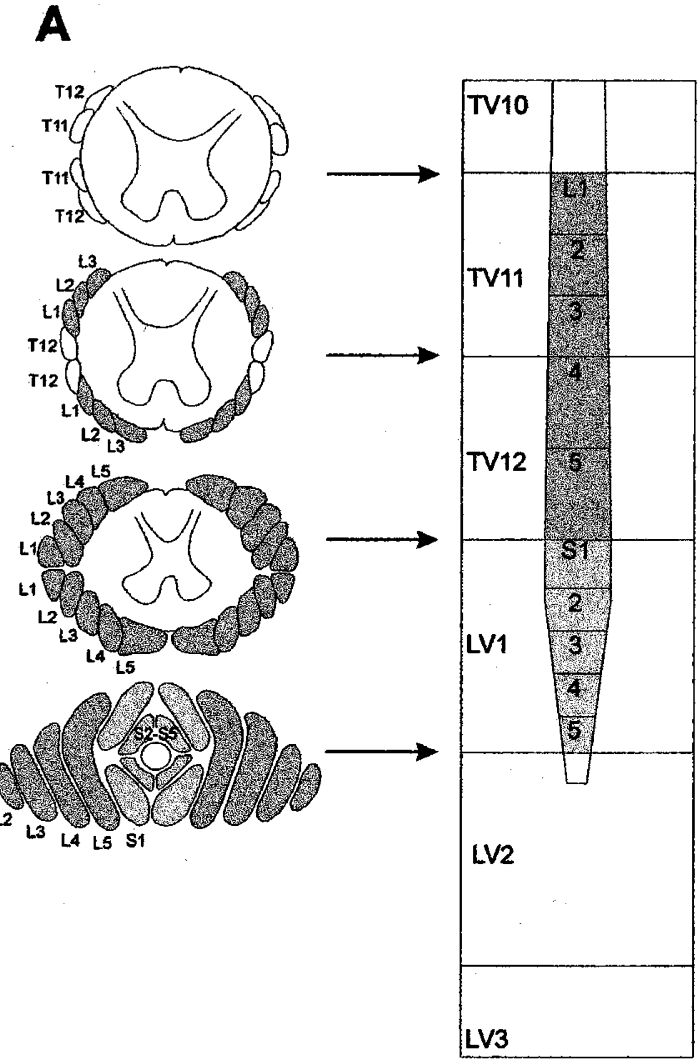

B

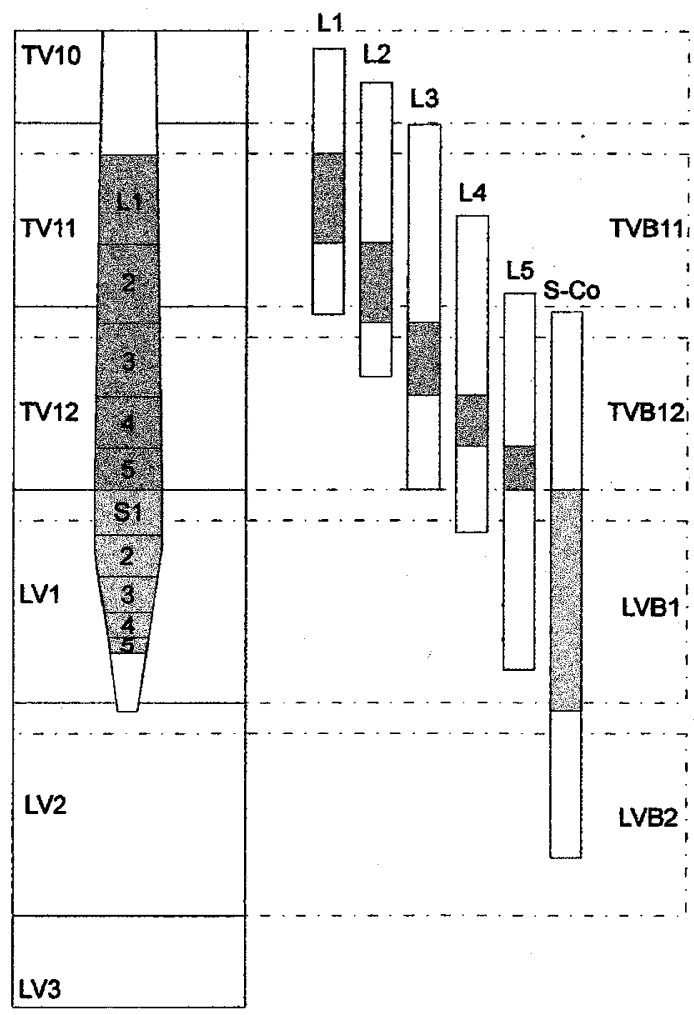

Figure 10 Relationship between spinal cord and vertebral segment levels. (A) 'Standard geometry' as used for the simulation. The arrangement of the spinal cord segments is constructed from spinal root fiber identification in four cross sections as reported by Wall et $a l^{15}$ : it is assumed that segments with fibers which are passing the observed cross-section are located completely above this cross section level. (B) Spinal cord geometry derived from Lang and Geisel data. ${ }^{22}$ Mean segment levels (gray bars) and their variations (white bars) at the right side are combined with mean segment lengths of Table 2 (gray area), TVB: thoracic vertebral bone, LVB: lumbar vertebral bone 
Relationship between fiber thresholds and muscle twitch responses

Action potentials initiated at low stimulation frequencies in the large dorsal root fibers seem to pass directly to the motor neuron of the same segment (monosynaptic pathway). The spinal cord segments are between 2 and $24 \mathrm{~mm}$ long. The segment separation causes the excitation of root fibers mainly because of their different distances to the cathode. According to the segmental arrangement of muscle innervation, and considering the mono-synaptic pathways, the recruitment order of dorsal roots is directly related to the sequence of responses of corresponding muscle groups (Figure 11). In our simulation the excitable neural structures of the spinal cord are reduced to 12 target neurons originating from three levels, representing L2-, L4- and S1-root fibers. Applying interpolation methods according to the scheme of Figure 2 allows to predict the thresholds for rootlets which were not considered in the simulation. This principle was used in the case study of Figure 11 in order to estimate the threshold for triceps surae, innervated by the segments L5, S1 and $\mathrm{S} 2$. The S1 target fiber (threshold $48 \mathrm{~V}$ ) will not contribute to any activation. However, the most cranial fibers that supply triceps surae arising from the L4/L5 borderline (see segmental arrangement of innervation in Figure 11) have an essentially lower threshold of about $6 \mathrm{~V}$ as found by interpolation (gray bar for TS, Figure 11). With the same method the thresholds as predicted by the target fibers (black bars) are corrected according to the gray areas by considering the complete innervation region of a muscle of interest.

We have used the same technique to predict muscle twitch thresholds for the four standard levels and both polarities (Figure 12). Of specific interest are those cases where the change of polarity activates an additional muscle group: In Figure 12A right, the cathode (upper contact) stimulates the L2 and L3 cord segments resulting in $\mathrm{Q}, \mathrm{A}$ and $\mathrm{H}$ responses. Changing polarity to $0+3-$ activates additionally the L4 and L5 segment, and the muscles TA and TS according to the segmental innervation arrangement, because now the cathode is at the L4 level. Moving the electrode $1 \mathrm{~cm}$ caudally (case $\mathrm{B}$, right) reduces the cathode distance to L4 for the $0-3+$ configuration and thereby allows the activation of TA at $3.5 \mathrm{~V}$; L5 is still beyond the excitation range, there is no TS response. However, the large shift of the cathode position stimulates also TS for the $0+3-$ polarity. The cathode is able to activate L4 and L5 posterior root fibers for both polarities when the electrode is positioned like Figure $12 \mathrm{C}$ or below; the resulting excitation patterns are similar. All presented calculations are based on activating the outer poles $(0,3)$ of the electrode. Smaller distances of the poles $($ eg $0-1+$ ) have a better focus effect but higher stimulus voltages are needed.

Comparison of patient muscle twitch data with the predicted muscle activation patterns of Figure 12 informs about individual spinal cord anatomy and helps to determine the position of the electrode relative to cord segments. We will discuss one example (Figure 13) where the muscle twitch responses of one patient recorded with surfaceelectrode polyelectromyography (pEMG) as reported in Murg et $a l^{2}$ is systematically compared with the four cases $\mathrm{A}-\mathrm{D}$ of Figure 12. Myograms of different responding muscle regions were systematically recorded while electrode voltage was increased in $1 \mathrm{~V}$ steps. At first we test if the number of activated muscle groups is reduced when polarity is changed from $0+3-$ to $0-3+$. Such a reduction implies an upper lumbar cord electrode position. Activation of all muscles for $0+3-$ but missing TA and TS for $0-3+$ indicates the correspondence of patients data

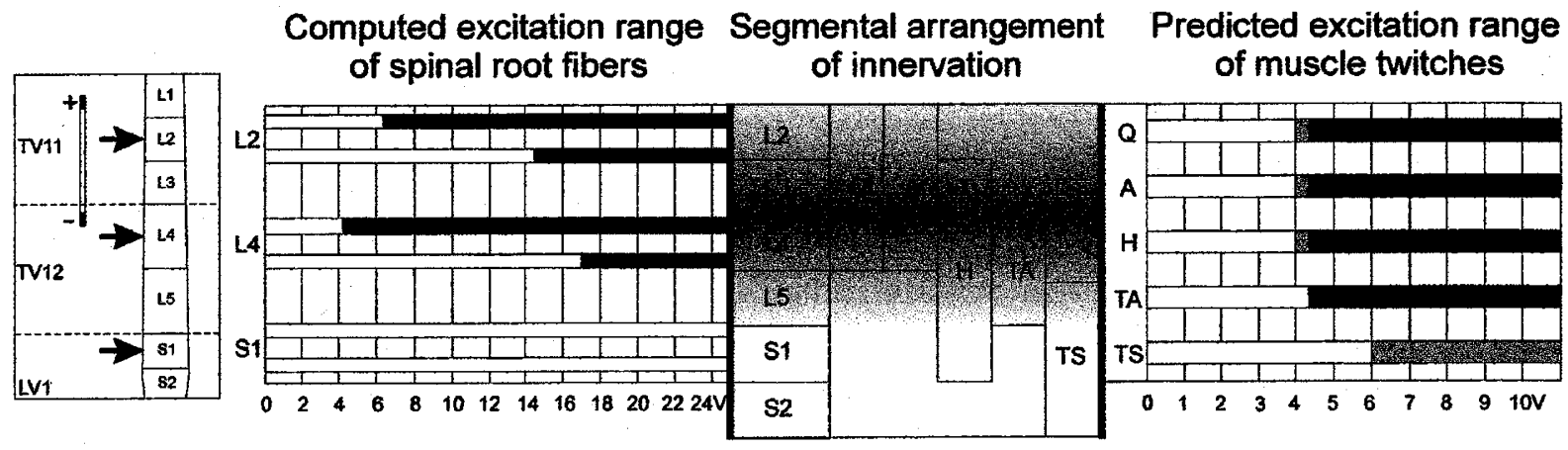

Figure 11 Relationship between computed spinal root fiber thresholds and muscle twitch thresholds. The gray levels in the shaded region indicate the excitability of the 'segmental arrangement of innervation'. The most excitable region for this position of the electrode (same case as Figure 6A) is at the L3/L4 border of the spinal cord. The black bars are computed regions of excitation when innervation is restricted to the standard fibers originating from three levels as indicated by the arrows (left picture). According to the innervation scheme (Table 14-1 from Westmoreland et al ${ }^{25}$ hamstring modified according to clinical observations $^{2}$ ) the L4 segment innervates the muscle groups Q, A, H and TA and therefore the black bars indicate the same threshold value of $4.3 \mathrm{~V}$ for these muscles. Thresholds for root fibers which were neglected in the simulation are found by interpolation and are used to refine the predicted muscle twitch thresholds by the gray bar. Q, quadriceps; A, adductor; H, hamstring; TA, tibialis anterior; TS, triceps surae 


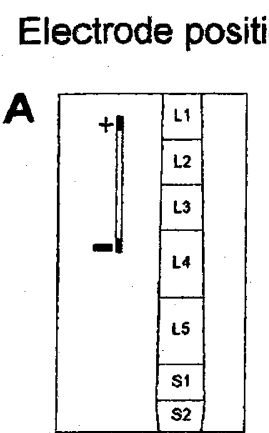

\section{Excitation range,} $0+3-$

\section{B}
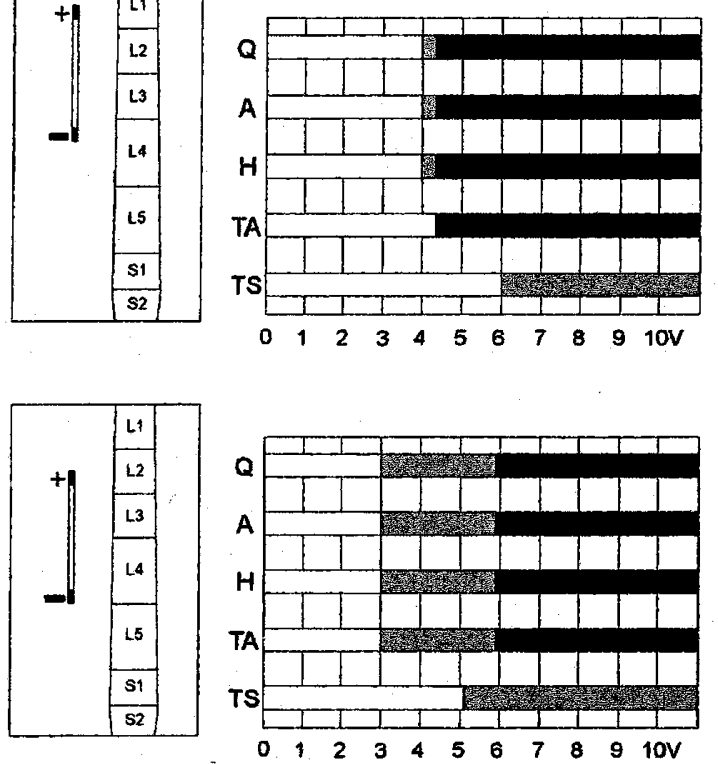

C
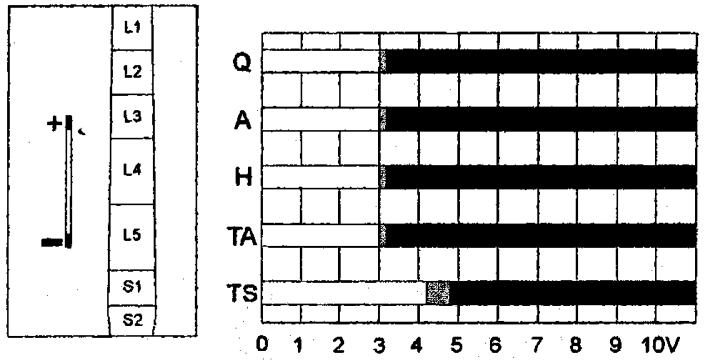

$\mathbf{D}$
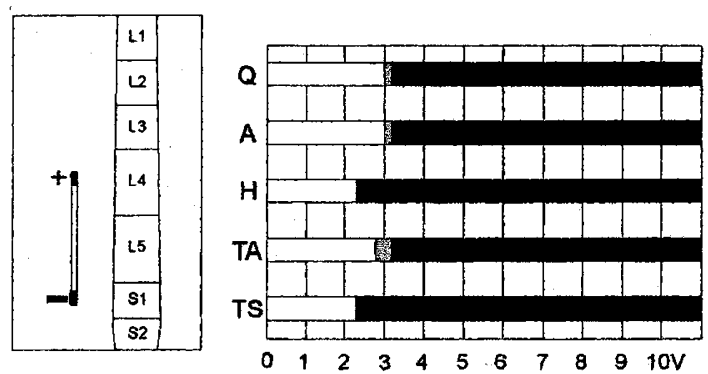

Figure 12 Predicted muscle twitch thresholds for electrode positions of Figure 6, method as in Figure 11

with case A. This means the lower contact is positioned at the L4 cord region. Considering additional information about the spinal cord termination level (MRI) and deviation from mean segment positions (Figure 10) we assume the number three contact to be at the lower part of L4. Note the high thresholds (small EMG amplitudes) for $0-3+$ which may be caused by a not optimally positioned upper contact (relative large resistance due to tissue placed between electrode and dura). Smaller resistance to the well conducting cerebrospinal fluid
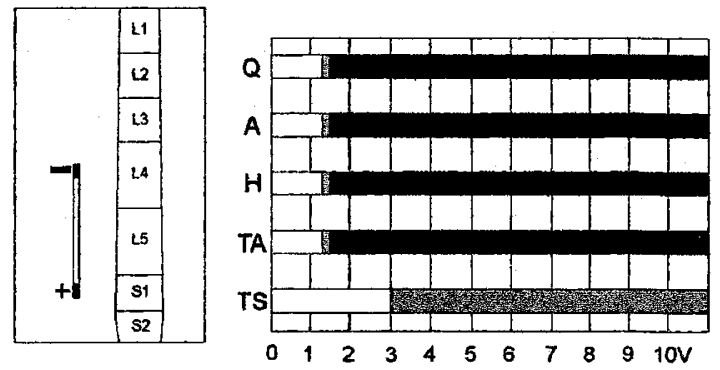

\section{Electrode position $0-3+$}
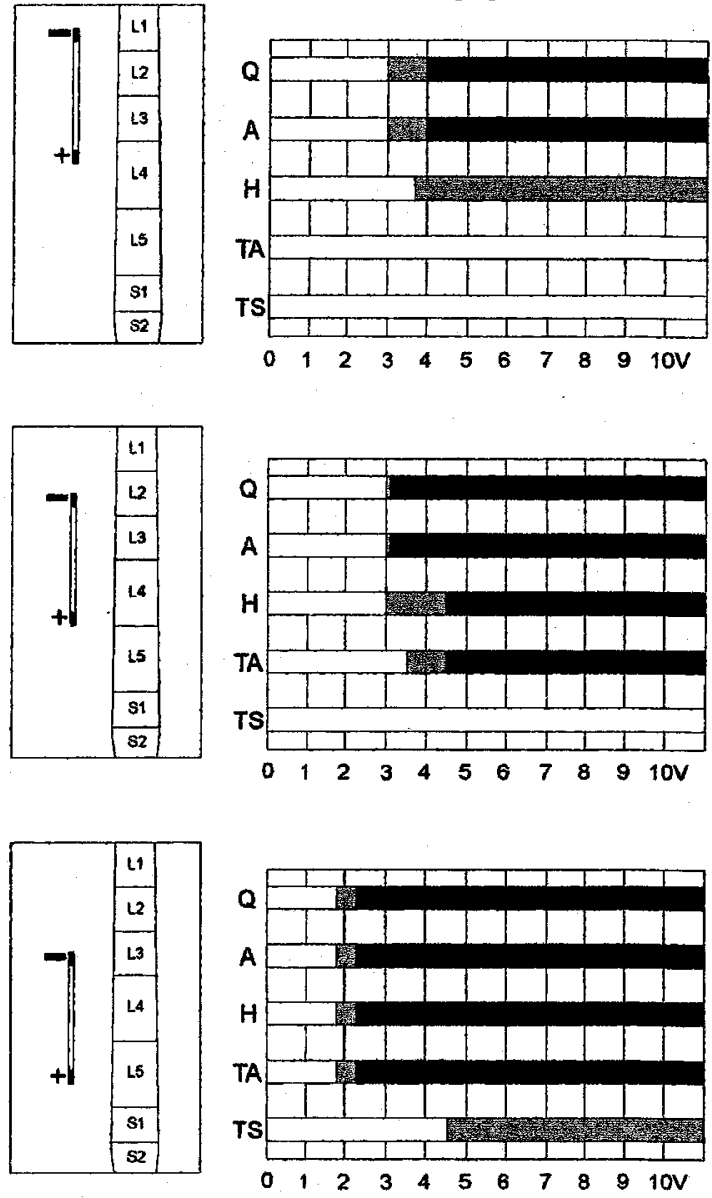

would shift the thresholds of all muscle groups to smaller values, TA response could be below $10 \mathrm{~V}$ and the position of the electrode relative to the L4 cord segment of this patient between the cases A and $\mathrm{B}$ of Figure 12 is also reasonable.

Factors limiting the accuracy of the simulations A well defined geometry of the volume conductor model is essential for the quality of the computations. A simplified finite element geometry was modeled to 
A Electrode position

relative to spinal cord Excitation range (computed)
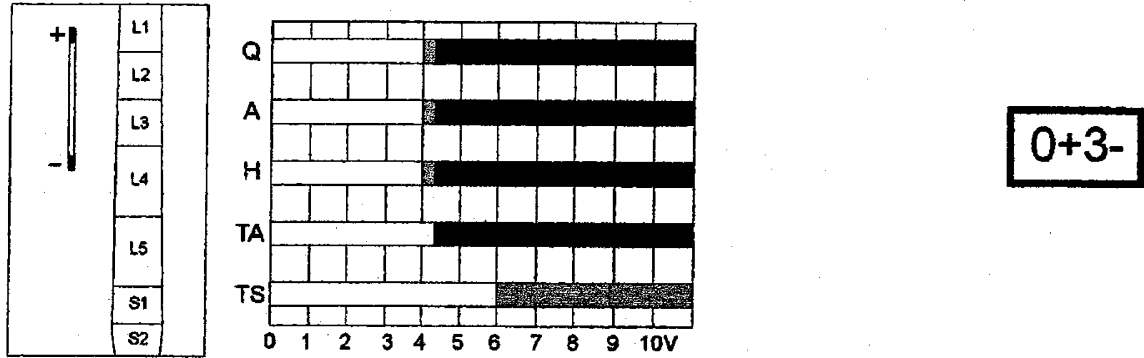

Electrode position

relative to vertebrae Excitation range (measured)
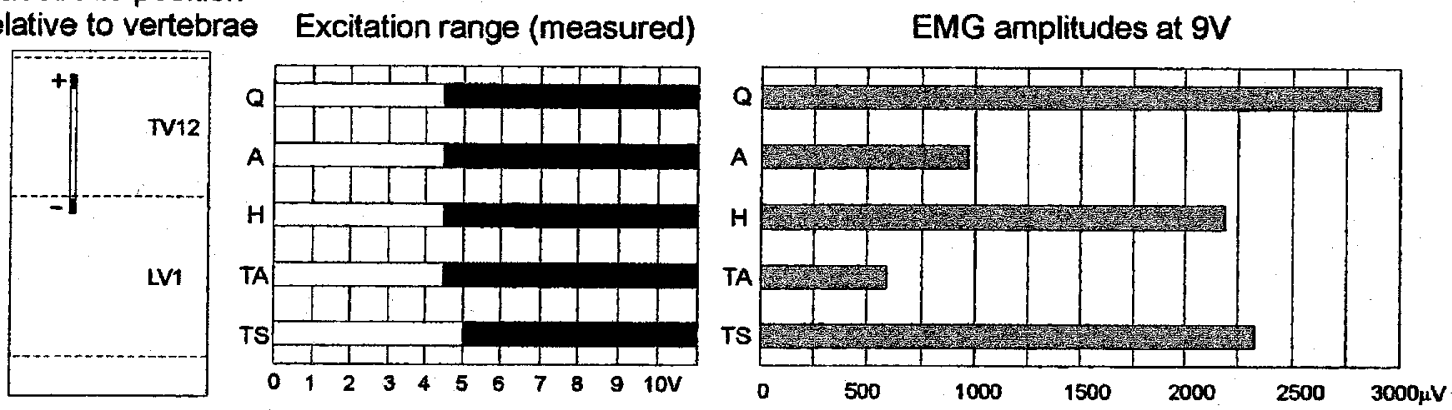

B Electrode position

relative to spinal cord Excitation range (computed)
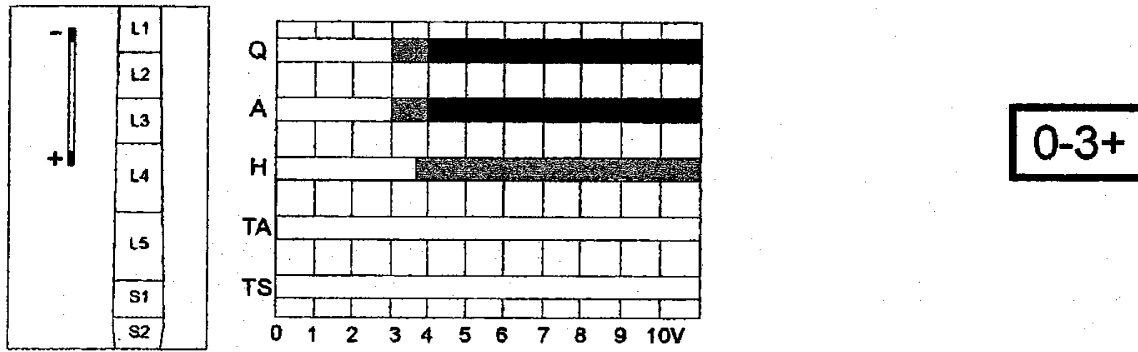

Electrode position relative to vertebrae

EMG amplitudes at $10 \mathrm{~V}$
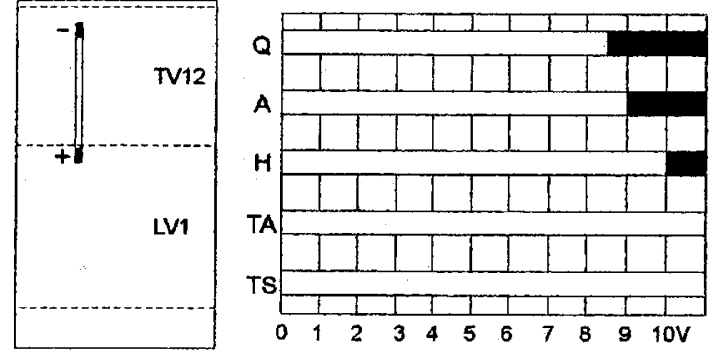

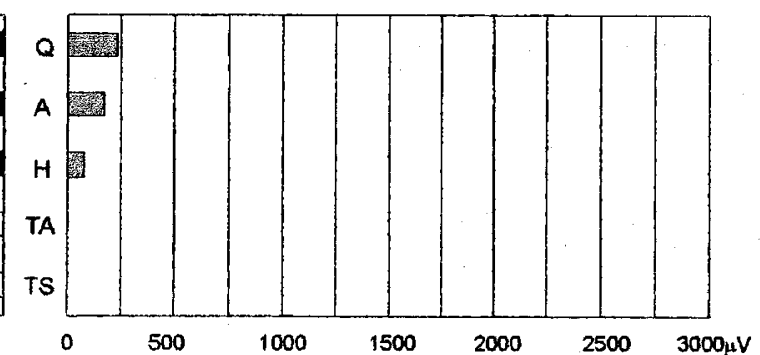

Figure 13 Comparison of computed and measured excitation patterns for a fixed electrode position. Electrode position relative to spinal cord and computed excitation range is same as in Figure 12A. Electrode position relative to vertebrae is according to $\mathrm{X}$-ray picture. White bar lengths are derived from $\mathrm{pEMG}$ protocol and correspond to the mean threshold values of the left and right muscle groups of a single patient. EMG amplitudes are mean values between left and right side, measured at the maximum voltage of $10 \mathrm{~V}$ or below if the stimulation was uncomfortable

represent the complex anatomical compartments. While the spinal canal is treated in detail in the simulation, only a coarse model of vertebral column and its surrounding layer is necessary for simulating the 
excitation of the neural structures with epidural electrodes. ${ }^{13}$ The small root filaments, immersed in the well-conducting cerebrospinal fluid, were not modeled as separate compartments in the volume conductor model. This model is valid under the assumption that the potential distribution in the cerebrospinal fluid surrounding the rootlets is not affected by the presence of these filaments. Note however that the lower part of the lumbar cord is surrounded by roots and the cord becomes completely covered by the roots below the twelfth thoracic vertebra $^{15}$ (see also the cross sections at the left part of Figure 10). Therefore, a refined model including the insulating property of the roots is assumed to show increase of threshold values for ventral roots and dorsal columns when the electrode is moved to the conus medullaris.

Most tissue conductivities are not exactly known. It is important to estimate the sensitivity of solutions to variations in tissue conductivity in normally accepted ranges. In another spinal cord study (unpublished results mentioned in Holsheimer ${ }^{13}$ ) these conductivities were increased and reduced by a factor of two, which is beyond the expected range. The worst case was a change in threshold stimulus of $19 \%$ for dorsal column fibers and $27 \%$ for dorsal root fibers.

In some clinical cases rather small threshold values were observed as muscle twitches in almost all of the lower limb muscles. These values are lower compared to our calculated data (Figure 12). An explanation is the small distance between electrode and roots by a local narrowing of the cerebrospinal fluid compartment, caused by the electrode pressing against the dura mater. In general we assume geometrical factors (eg size of compartments, position of the spinal cord segments relative to the vertebral levels, the trajectories of the roots) to have more influence on the observed range of threshold values than individual differences in the conductances.

\section{Conclusions}

Computer simulations demonstrate that thick dorsal root fibers are the most excitable structures for lumbar spinal cord stimulation with posterior epidural electrodes. The recruitment order of lower limb muscles essentially depends on the level of the cathode: For cathode positions at L4 cord level and below all observed muscles $(\mathrm{Q}, \mathrm{A}, \mathrm{H}, \mathrm{TA}, \mathrm{TS})$ are expected to respond within a range of about 3-6 V (Figure 12). Changing the polarity has small influence on the pattern of muscle activation for sacral and low lumbar electrode positions, ie both poles are below L3 cord level. When the complete electrode is moved superior to L5, change of polarity (a $2.7 \mathrm{~cm}$ shift of cathode center in the simulated cases) causes systematical loss of muscle group responses in a characteristic way. This phenomenon can be used to identify the electrode position relative to the spinal cord segment for levels
L2-L5: as shown in Figure 12B loss of the TS response (change from $0+3-$ to $0-3+$ ) indicates that the lower contact is located close to the L4/L5 border. In an analogous way, additional loss of TA response is a characteristic hint for a $1 \mathrm{~cm}$ higher electrode position, ie close to the border region L3/L4 (Figure 12A). When the lower contact is at upper L2 cord level $0+3$ - will activate $\mathrm{Q}, \mathrm{A}$ and possibly $\mathrm{H}$ whereas at changed polarity no muscle groups will be recruited. This method for segment localization works with monophasic pulses and - as in our clinical applications - with biphasic stimuli when a long second phase with small amplitude is used for charge compensation. This method is not suited for biphasic signals with phases of the same duration and amplitude (comp. Figure 8).

\section{Acknowledgements}

We thank Dr F Gielen from Medtronics for saline tank measurements, Dr D Prayer from Vienna University Hospital for MRI support, Dr M Pinter and Dr M Murg for many helpful discussions, and Dr L-E Larsson from Stockholm for valuable suggestions to improve the manuscript.

\section{References}

1 Dimitrijevic MR, Gerasimenko Y, Pinter MM. Evidence for a spinal central pattern generator in humans. Ann NY Acad Sci 1998; 860: $360-376$.

2 Murg M, Binder H, Dimitrijevic MR. Epidural electric stimulation of posterior structures of the human lumbar spinal cord: 1. Muscle twitches - a functional method to define the site of stimulation. Spinal Cord 2000, (in press).

3 Pinter MM, Gerstenbrand F, Dimitrijevic MR. Epidural electric stimulation of posterior structures of the human lumbar spinal cord: 3. Control of spasticity. Spinal Cord 2000, (in press).

4 Rattay F. Electrical nerve stimulation: theory, experiments and applications. Springer. Wien New York 1990.

5 Rattay F. The basic mechanism for the electrical stimulation of the nervous system. Neuroscience 1999; 89: 335-346.

6 Minassian K. Excitation of lower spinal cord structures with implanted electrodes: 3D finite element analysis and simulation of neural responses. Diploma thesis. Vienna University of Technology. 2000

7 Porter R. Focal stimulation of hypoglossal neurons in the cat. $J$ Physiol 1963; 169: 630-640.

8 Rattay F. Analysis of the electrical excitation of CNS neurons. IEEE-Trans Biomed Eng 1998; 45: 766-772.

9 Coburn B, Sin WK. A theoretical study of epidural electrical stimulation of the spinal cord - Part I: Finite element analysis of stimulus fields. IEEE Trans Biomed Eng 1985; 32: 971 - 977.

10 Coburn B. A theoretical study of epidural electrical stimulation of the spinal cord - Part II: Effects on long myelinated fibers. IEEE Tranas Biomed Eng 1985; 32: 978 - 986.

11 Holsheimer J, Struijk JJ. How do geometric factors influence epidural spinal cord stimulation? A quantitative analysis by computer modeling. Stereotact Funct Neurosurg 1991; 56: $234-$ 249.

12 Struijk JJ, Holsheimer J, Boom HB. Excitation of dorsal root fibers in spinal cord stimulation: A theoretical study. IEEE Trans Biomed Eng 1993; 40: 632-639.

13 Holsheimer J. Computer modelling of spinal cord stimulation and its contribution to therapeutic efficacy. Spinal Cord 1998; 36: $531-540$. 
14 Szentágothai-Schimert. Bedeutung des Faserkalibers und der Markscheidendicke im Zentralnervensystem. Zeitschrift $f$ Anatomie u Entwicklungsgeschichte 1941; 111: 201-223.

15 Wall EJ, Cohen MS, Abitbol JJ, Garfin SR. Organization of intrathecal nerve roots at the level of the conus medullaris. $J$ Bone Joint Surg [Am] 1990; 72: 1495-1499.

16 Kameyama T, Hashizume Y, Sobue G. Morphologic features of the normal human cadaveric spinal cord. Spine 1996; 21: 12851290.

17 Bulling A et al. Body Explorer 2.0, An interactive program on the cross-sectional anatomy of the visible human male. Springer Verlag Heidelberg 1997.

18 Geddes LA, Baker LE. The specific resistance of biological material a compendium of data for the biomedical engineer and physiologist. Med Biol Eng 1967; 5: 271 -293.

19 Struijk JJ et al. Paresthesia thresholds in spinal cord stimulation: A comparison of theoretical results with clinical data. IEEE Trans Rehab Eng 1993; 1: 101 - 108.
20 Schwan HP, Kay CF. The conductivity of living tissues. Ann NY Acad Sci 1957; 65: 1007-1013.

21 Sweeney JD, Mortimer JT, Durand D. Modeling of mammalian myelinated nerve for functional neuromuscular electrostimulation. Proc 9th Ann Conf, IEEE-EMBS 1987, pp 1577-1578.

22 Lang J, Geisel U. Über den lombsakralen teil des durasackes und die topographie seines inhalts. Morphol Med 1983; 3: 27-46.

23 Diem MP. Vergleichende längenmessungen an vorderen nervenwurzeln bei neugeborenen und erwachsenen. Med Thesis Zürich 1980.

24 Lüderitz C. Über das rückenmarksegment. Ein beitrag zur morphologie und histologie des rückenmarks. Arch Anat Physiol $1881 ; 423-495$.

25 Westmoreland BF et al. Medical Neurosciences. 3rd edn. Little, Brown and Company. Boston 1994 